Document downloaded from:

http://hdl.handle.net/10251/55795

This paper must be cited as:

Selga, J.; Rodríguez Pérez, AM.; Boria Esbert, VE.; Martín, F. (2013). Synthesis of splitrings-based artificial transmission lines through a new two-step, fast converging, and robust aggressive space mapping (ASM) algorithm. IEEE Transactions on Microwave Theory and Techniques. 61(6):2295-2308. doi:10.1109/TMTT.2013.2259254.

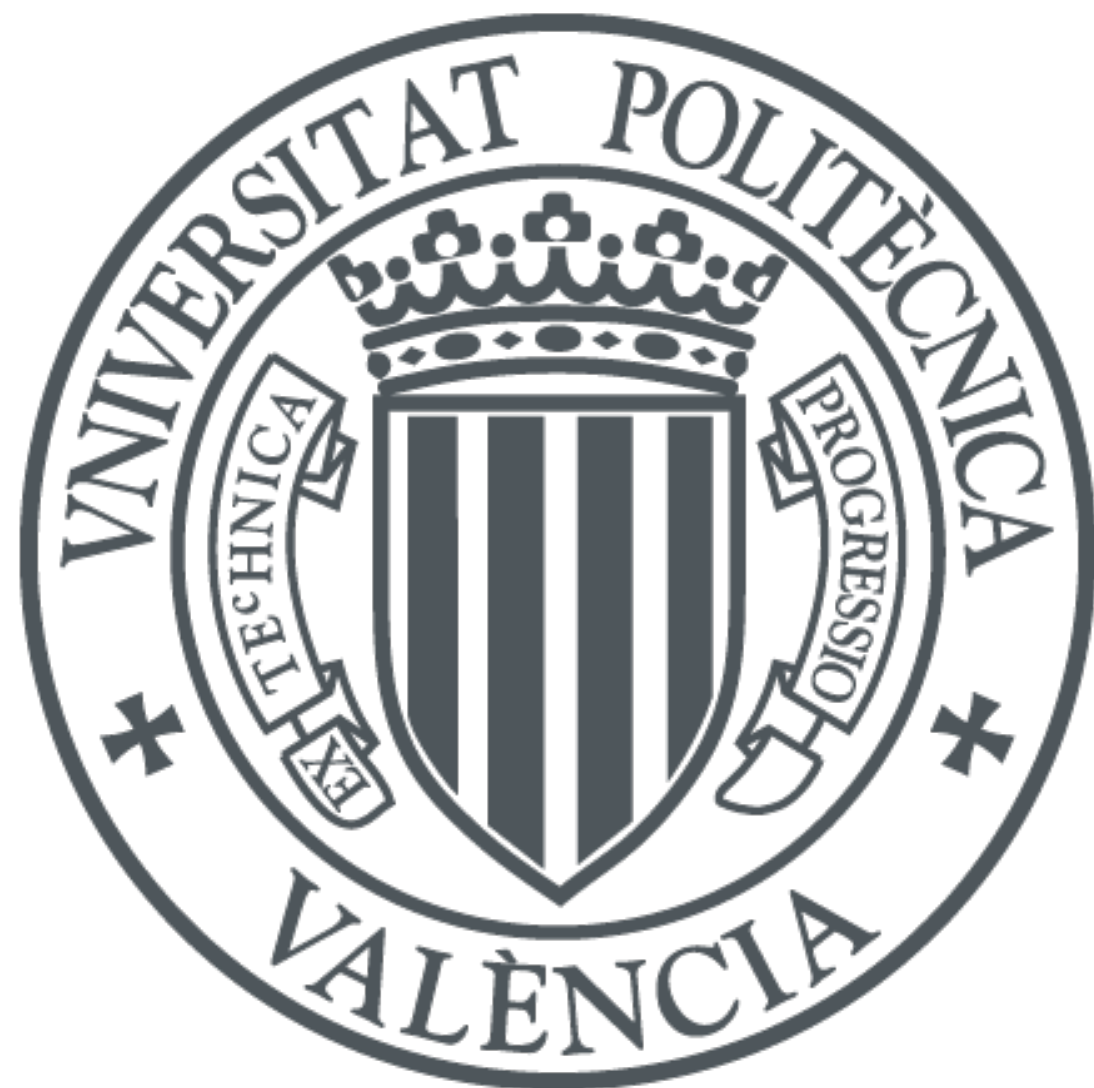

The final publication is available at

http://dx.doi.org/10.1109/TMTT.2013.2259254

Copyright Institute of Electrical and Electronics Engineers (IEEE)

Additional Information

(C) 2013 IEEE. Personal use of this material is permitted. Permission from IEEE must be obtained for all other uses, in any current or future media, including reprinting/republishing this material for advertising or promotional purposes, creating new collective works, for resale or redistribution to servers or lists, or reuse of any copyrighted component of this work in other works. 


\title{
Synthesis of Split Rings based Artificial Transmission Lines through a New Two-Step, Fast Converging, and Robust Aggressive Space Mapping (ASM) Algorithm
}

\author{
Jordi Selga, Ana Rodríguez, Vicente E. Boria, Senior Member IEEE, and Ferran Martín, Fellow IEEE
}

\begin{abstract}
This paper is focused on the synthesis of artificial transmission lines based on complementary split ring resonators (CSRRs). The considered structures are microstrip lines with CSRRs etched in the ground plane and microstrip lines loaded with both CSRRs and series capacitive gaps. An aggressive space mapping (ASM) optimization algorithm, able to automatically generate the layout of these artificial lines, has been developed. The tool has been optimized in order to achieve fast convergence and to provide accurate results. The main relevant aspects of the proposed algorithm (based on a novel two-step ASM optimization approach) are: (i) the capability to provide the implementable circuit elements of the equivalent circuit model of the considered artificial lines, and (ii) the ability to converge in few (un-precedent) iteration steps, due to a new procedure to generate the initial layouts (which are very close to the final ones). First, the software is tested through the synthesis of several CSRR-based microstrip lines, and then some practical application examples of such artificial lines are reported to illustrate the potential of the proposed synthesis tool.
\end{abstract}

Index Terms- Space mapping, complementary split ring resonator (CSRR), artificial transmission lines, metamaterial transmission lines, microstrip.

\section{INTRODUCTION}

The topic of artificial transmission lines has attracted much attention in the last decade and opened a path to a new type of artificial transmission lines based on metamaterial concepts [1]-[6]. Metamaterial-based (or inspired) transmission lines consist of a host line loaded with reactive elements (inductors, capacitors, resonators, or a combination of them). Such lines exhibit controllable dispersion and characteristic impedance, this being the main relevant advantage as compared to conventional lines, or to other artificial transmission lines. In conventional lines, the line impedance can be tailored to some extent through the geometry; however, the phase constant (for a given substrate) is barely dependent on the geometry. In certain artificial transmission lines, such as slow wave

This work has been supported by MICIIN-Spain (projects TEC201017512 METATRANSFER, TEC2010-21520-C04-01, CONSOLIDER EMET CSD2008-00066, and grant AP2008-04707), Generalitat de Catalunya (project 2009SGR-421), and MITyC-Spain (project TSI-020100-2010-169 METASINTESIS).

J. Selga and F. Martín are with GEMMA/CIMITEC, Departament d'Enginyeria Electrònica, Universitat Autònoma de Barcelona, 08193 Bellaterra, Spain. E-mail: Ferran.Martin@uab.es.

A. Rodríguez and V.E. Boria are with Departamento de ComunicacionesiTEAM, Universidad Politécnica de Valencia, 46022 Valencia, Spain. Email: vboria@dcom.upv.es. transmission lines, a host line is periodically loaded with capacitors, inductors or by means of semi-lumped reactive elements, which effectively increase the line capacitance or inductance, and hence reduce the phase velocity [7]-[9].

In metamaterial transmission lines, the presence of reactive elements increases the design flexibility of the lines. Thus, the characteristic impedance and the phase constant (as well as the phase and group velocities) can be engineered in order to fulfill line specifications or functionalities not achieved before. Size reduction [10],[11], broadband [10],[12]-[16] and multiband operation [17]-[21], leaky wave structures with steerable radiation beams [22],[23], or novel broadband filters and diplexers with spurious suppression [24]-[26], (including tunable filters [27]) are some of the applications of metamaterial transmission lines. In these applications, device design is based on both impedance and dispersion engineering.

The early metamaterial transmission lines were implemented by loading a host line with series capacitors and shunt inductors [2]-[4]. At low frequencies, the loading elements are dominant, and the structure behaves as a backward wave transmission line. In this regime, the phase and group velocities are anti-parallel and the structure supports backward waves, or, in other words, it exhibits a lefthanded behavior. At higher frequencies, the line inductance and capacitance are dominant, and the propagation is forward (or right-handed). Therefore, these CL-loaded lines exhibit composite right/left handed (CRLH) wave propagation [28],[29]. Such CRLH behavior can also be achieved by means of metamaterial transmission lines based on electrically small resonators, such as split ring resonators (SRRs) [30] or complementary split ring resonators (CSRRs) [31]. In the latter case, which is the relevant one for the purposes of the present paper, a host line, typically (although not exclusively) a microstrip line, is loaded with CSRRs (etched in the ground plane beneath the conductor strip) and series capacitive gaps on top of the CSRRs. These CSRRbased lines do also exhibit a CRLH behavior. However, the circuit model, depicted in Fig. 1(a), reveals the presence of a coupling capacitance, $C$, between the CSRR (modeled by $L_{c}$ and $C_{c}$ ) and the line, responsible for the presence of a transmission zero. $L$ is the line capacitance and $C_{g}$ is related to the gap capacitance. In fact, assuming that the series gap can be modeled by a $\pi$-network with series capacitance $C_{s}$ and shunt (fringing) capacitance $C_{f}$, it follows [32]:

$$
2 C_{g}=2 C_{s}+C_{p a r}
$$




$$
C=\frac{C_{p a r}\left(2 C_{s}+C_{p a r}\right)}{C_{s}}
$$

with $C_{p a r}=C_{f}+C_{L}, C_{L}$ being the line capacitance.

The frequency response of CL-loaded and CSRR-based CRLH lines exhibits a pass band in the left-handed and the right-handed regions, and these two bands merge if the CRLH line is balanced, that is, if the series and shunt resonance frequencies are identical. By removing the gap, the pass band behavior of CSRR-based lines switches to a stop band, caused by the presence of the transmission zero (the circuit model for these structures is also depicted in Fig. 1b). This stop band functionality is of interest for the implementation of notch filters and band-stop filters [33].

Despite the numerous applications and advantages of CSRR-based artificial lines, a weak point towards the further penetration of these lines into the market concerns their synthesis. In this regard, recent efforts have been made by the authors in order to implement efficient algorithms for layout generation. Specifically, in [34] and [35], the potential of space mapping optimization for the synthesis of CSRR-based lines acting as stop band structures (i.e., without the presence of the gaps) was demonstrated. In the present paper, we extend the space mapping optimization to the synthesis of CSRR and gap loaded lines. Moreover, as compared to [34] and [35], we introduce several improvements that provide faster convergence and major robustness to the algorithm, and a method able to give the implementable element values of the equivalent circuit model.


Fig.1. Typical topologies and circuit models (unit cell) of a CSRR-loaded microstrip line with (a) and without (b) series gap. The ground plane, where the CSRR are etched, is depicted in grey.

This paper is organized as follows. In section II, the general formulation of ASM is provided for completeness and for better comprehension of the paper. Section III is focused on the application of ASM optimization to the synthesis of CSRR-based lines, where the new two-step ASM algorithm (based on a novel method to determine the initial geometry) is presented. In section IV, the new two-step ASM-based tool is applied to the synthesis of practical microwave circuits, including a band-stop filter and a dual-band power divider. Finally, the main conclusions are highlighted in section V.

\section{GENERAL FORMULATION OF AGGRESSIVE SPACE MAPPING}

(ASM)

Space mapping is a technique extensively used in the optimized design process of microwave devices, which makes proper use of two simulation spaces [36]-[38]. In the optimization space, $\mathbf{X}_{\mathbf{c}}$, the variables are linked to a coarse model, which is simple and computationally efficient, although not accurate. On the other hand, the variables corresponding to the validation space, $\mathbf{X}_{\mathbf{f}}$, are linked to a fine model, typically more complex and $\mathrm{CPU}$ intensive, but significantly more precise. In each space, we can define a vector containing the different model parameters. Let us denote such vectors as $\mathbf{x}_{\mathbf{f}}$ and $\mathbf{x}_{\mathbf{c}}$ for the fine model and coarse model parameters, respectively. Using the same nomenclature, $\mathbf{R}_{\mathbf{f}}\left(\mathbf{x}_{\mathbf{f}}\right)$ and $\mathbf{R}_{\mathbf{c}}\left(\mathbf{x}_{\mathbf{c}}\right)$ will denote the responses of the fine and coarse models, respectively. For microwave applications, the model response is related to the evaluation of the device behaviour, e.g., a scattering parameter, such as $\left|S_{11}\right|$ or $\left|S_{21}\right|$, computed in a certain frequency range.

The key idea behind the space mapping algorithm is to generate an appropriate parameter transformation

$$
\mathbf{x}_{\mathrm{c}}=\mathbf{P}\left(\mathbf{x}_{\mathbf{f}}\right)
$$

mapping the fine model parameter space to the coarse model parameter space such that

$$
\left\|\mathbf{R}_{\mathbf{f}}\left(\mathbf{x}_{\mathbf{f}}\right)-\mathbf{R}_{\mathbf{c}}\left(\mathbf{x}_{\mathbf{c}}\right)\right\| \leq \eta
$$

in some predefined region, $\|\cdot\|$ being a certain suitable norm and $\eta$ a small positive number close to zero. If $\mathbf{P}$ is invertible, then the inverse transformation:

$$
\mathbf{x}_{\mathbf{f}}=\mathbf{P}^{-1}\left(\mathbf{x}_{\mathbf{c}}^{*}\right)
$$

is used to find the fine model solution, which is the image of the coarse model solution, $\mathbf{x}_{\mathbf{c}}{ }^{*}$, that gives the target response, $\mathbf{R}_{\mathbf{c}}\left(\mathbf{x}_{\mathbf{c}}{ }^{*}\right)$.

The determination of $\mathbf{P}$ according to the procedure reported in [36] follows an iterative process that is rather inefficient. However, the efficiency of the method can be improved by introducing a quasi-Newton type iteration [37]. This method aggressively exploits each fine model EM analysis with the result of a faster convergence. Hence, the new approach was called aggressive space mapping (ASM) [37], and it is the optimization procedure considered in this work. Essentially, the goal in ASM is to solve the following set of non-linear equations:

$$
\mathbf{f}\left(\mathbf{x}_{\mathbf{f}}\right)=\mathbf{P}\left(\mathbf{x}_{\mathbf{f}}\right)-\mathbf{x}_{\mathbf{c}}^{*}=0
$$

For a better understanding of the iterative optimization process, a superscript is added to the notation that actually indicates the iteration number. Hence, let us assume that $\mathbf{x}_{\mathbf{f}}{ }^{(j)}$ is the $j$-th approximation to the solution of (6) and $\mathbf{f}^{(j)}$ the error function corresponding to $\mathbf{f}\left(\mathbf{x}_{\mathbf{f}}{ }^{(j)}\right)$. The next vector of the iterative process $\mathbf{x}_{\mathbf{f}}^{(j+1)}$ is obtained by a quasi-Newton iteration according to

$$
\mathbf{x}_{\mathbf{f}}^{(j+1)}=\mathbf{x}_{\mathbf{f}}^{(j)}+\mathbf{h}^{(j)}
$$

where $\mathbf{h}^{(j)}$ is given by:

$$
\mathbf{h}^{(j)}=-\left(\mathbf{B}^{(j)}\right)^{-1} \mathbf{f}^{(j)}
$$

and $\mathbf{B}^{(j)}$ is an approach to the Broyden matrix [37]: 


$$
\mathbf{B}^{(j+1)}=\mathbf{B}^{(j)}+\frac{\mathbf{f}^{(j+1)} \mathbf{h}^{(j) T}}{\mathbf{h}^{(j) T} \mathbf{h}^{(j)}}
$$

which is also updated at each iterative step. In (9), $\mathbf{f}^{(j+l)}$ is obtained by evaluating (6) using a certain parameter extraction method providing the coarse model parameters from the fine model parameters, and the super-index $T$ stands for transpose.

The implementation of the ASM algorithm is well reported in [37]. If the fine and coarse models involve the same space parameters, then the first vector in the fine space is typically set equal to the target vector in the coarse space, and the Broyden matrix is initialized by forcing it to be the identity. However, since this is not the case we are considering in this paper, we have followed a different approach (section III.C).

\section{SYNTHESIS OF CSRR-BASED ARTIFICIAL TRANSMISSION LINES BY MEANS OF THE NOVEL TWO-STEP ASM APPROACH}

From now on, and for clear distinction, microstrip lines loaded only with CSRRs will be called CSRR-loaded metalines, and those lines loaded with both CSRRs and series gaps will be designated as CSRR-gap-loaded metalines. Let us first describe the two spaces involved in the proposed ASM algorithm for both line types. The optimization space, $\mathbf{X}_{\mathbf{c}}$, is the circuit model, and the space variables are the circuit elements appearing in the equivalent circuits of Figs. 1(a) and (b) for the CSRR-gap- and CSRR-loaded lines, respectively. The validation space, $\mathbf{X}_{\mathbf{f}}$, is the electromagnetic (EM) model of the physical structure that provides the frequency response from the geometry and substrate parameters. The substrate parameters will be set to certain values and therefore these parameters are not considered as variables of the fine model. The chosen substrate is the Rogers RO3010 with dielectric constant $\varepsilon_{r}=10.2$ and thickness either $h=1.27 \mathrm{~mm}$ or $h=0.635 \mathrm{~mm}$ (losses are not considered in the EM model). Some constraints will be applied to the geometry parameters in order to reduce the degrees of freedom and thus work with the same number of variables in both spaces, as it was done in [34] (the matrix $\mathbf{B}$ is thus square and invertible, and the computational effort is minimized).

The most relevant difference between the ASM algorithm reported in this paper, with regard to the one reported in [34], is the determination of the initial layout. With the new proposed strategy, the initial estimated layout (i.e. $\mathbf{x}_{\mathbf{f}}^{(0)}$ ) is already very close to the optimum final solution $\mathbf{x}_{\mathbf{f}}{ }^{*}$, leading to a fast and very soft convergence rate of the whole synthesis procedure. Moreover, our previous implementations of ASM algorithms [34],[35],[39] are not able to guarantee if there is a mapping between the optimal coarse model solution (i.e., a set of circuit parameters corresponding to the target response) and a fine model point providing a physically implementable layout. In this paper, we overcome this practical limitation through the determination of a convergence region in the coarse model space. Hence, we will first present this novel strategy for the determination of the convergence regions, which involves a pre-optimization ASM scheme. For this reason, our proposed synthesis tool is based on a two-step ASM approach (see Fig. 2).

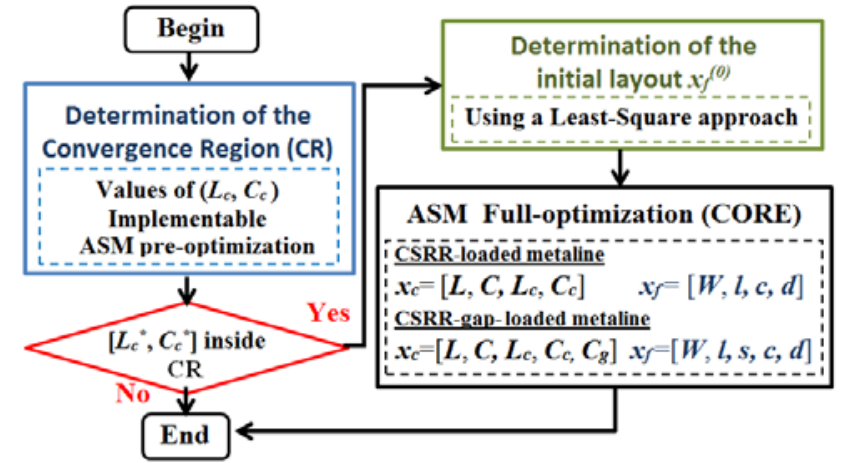

Fig.2. Schematic of the new proposed two-step ASM algorithm (see the text for better comprehension).

\section{A. Determination of the Convergence Region: Pre- optimization ASM Algorithm}

The implementable layouts of CSRR based lines are limited to certain combinations of the element values of the circuit models. Despite some efforts having made by means of parametric analysis [32], there is not yet a systematic procedure to determine whether a given combination of circuit model elements can be translated to an implementable geometry or not. This is a fundamental problem to solve in order to implement a practical synthesis tool, and it is the subject of the present subsection.

Let us first consider the case of CSRR-loaded metalines. The geometrical variables are the microstrip line length, $l$, and width, $W$, and the width, $c$, and separation, $d$, of the slot rings (see Fig. 1b). In order to deal with the same number of variables in the coarse and fine model spaces, we consider $s_{\text {split }}=c$ and $l=2 r_{\text {ext }}$, where $r_{\text {ext }}$ is the external radius of the CSRR. This choice is justified to avoid distributed effects that may appear if $l>2 r_{e x t}$ and are not accounted for by the circuit model and to ensure that the ports of the structure are accessible.

Given a set of circuit (target) variables, $L^{*}, C^{*}, L_{c}{ }^{*}$ and $C_{c}{ }^{*}$, a procedure to determine if this set of variables has a physically implementable layout is needed. According to the circuit model, $L^{*}$ and $C^{*}$ are physically realizable if these values are not too extreme and a microstrip line with reasonable width and length results. The important aspect is thus, given a pair of implementable values of $L^{*}$ and $C^{*}$, to determine the convergence region for the circuit values modeling the CSRR $\left(L_{c}\right.$ and $C_{c}$ ). So the strategy consists on calculating the line geometry that provides the element values $L^{*}$ and $C^{*}$ under four different scenarios corresponding to the extreme values of $c$ and $d$, namely, $c_{\min }-d_{\min }, c_{\max }-d_{\max }, c_{\min }-d_{\max }$, and $c_{\max }-d_{\min }$. The parameters $c_{\min }$ and $d_{\min }$ are the minimum achievable slot and strip widths, respectively, with the technology in use (we have set $c_{\min }=d_{\min }=0.15 \mathrm{~mm}$ in this work, unless otherwise specified). On the other hand, $c_{\max }$ and $d_{\max }$ are set to a reasonable (maximum) value that guarantees the validity of the model in the frequency region of interest. It has been found that for values exceeding $0.4-0.5 \mathrm{~mm}$, the coupling between the slot rings is very limited and hence we have set $c_{\max }=d_{\max }=0.4-0.5 \mathrm{~mm}$ (larger values expand the convergence region, but at the expense of less accuracy in the final solutions). 
For each case, the single geometrical variables are $l$ and $W$ ( $c$ and $d$ are fixed and $r_{\text {ext }}=l / 2$ ). These variables must be optimized with the goal of recovering the $L$ and $C$ values corresponding to the target $\left(L^{*}\right.$ and $\left.C^{*}\right)$. The extraction of the elements of the circuit model from the EM simulation of the CSRR-loaded line layout (which is a straightforward technique) has been reported before [35], but it is reproduced in Appendix A for completeness (parameter extraction is a fundamental building block of the proposed ASM algorithm).

For the determination of $l$ and $W$, we do also consider an ASM optimization scheme, which is very simple since only two variables in each space are involved. The initial values of $l$ and $W$ are obtained from the characteristic impedance and phase constant resulting from $L^{*}$ and $C^{*}$, with the help of a transmission line calculator. With the resulting line geometry, we extract the parameters of the circuit model and also initialize the Broyden matrix (the initiation of the Broyden matrix follows a similar approach to that corresponding to the core ASM algorithm, which is reported in Section III.C). Then the process iterates until convergence is achieved. In this pre-optimization ASM process, the stopping criterion is usually tighter than the one considered for the full-ASM optimization, i.e. smaller values of the error functions are forced. This more restrictive criterion is chosen not only to accurately determine the vertices of the convergence region, but also to obtain a better estimate of the initial layout of the core ASM algorithm (however this does not require much computational effort due to the very small number of terms -coarse model parameters- involved in the error calculation).

Once the $l$ and $W$ values corresponding to the target $L^{*}$ and $C^{*}$ for a given $c$ and $d$ combination (for instance $c_{\text {min }}-d_{\text {min }}$ ) are found, the whole geometry is known, and the element values $L_{c}$ and $C_{c}$ can be obtained by means of the parameter extractor. These element values $\left(L_{c}\right.$ and $\left.C_{c}\right)$ correspond to the considered CSRR geometry $\left(c_{\min }-d_{\min }\right)$, and actually define the

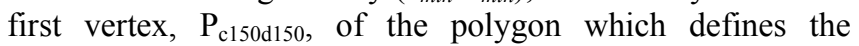
convergence region, see Fig. 3. Notice that the nomenclature used for identifying the vertices indicates (subscript) the values of $c$ and $d$ in microns.

Then, the same process is repeated for obtaining the next points, i.e, the $L_{c}$ and $C_{c}$ pairs corresponding to $c_{\max }-d_{\max }, c_{\min }{ }^{-}$ $d_{\max }$, and $c_{\max }-d_{\min }$ with the target $L^{*}$ and $C^{*}$ (for these cases the initial values of $l$ and $W$ are set to the solutions of the previous vertex, since it provides a much better approaches to the optimal final solution). As a result, we obtain a set of points in the coarse model sub-space of $L_{c}$ and $C_{c}$ that defines a polygon (see Fig. 3). This polygon is a rough estimate for the region of convergence in the $L_{c}-C_{c}$ subspace, for the target values $L^{*}$ and $C^{*}$. The criterion to decide if the target element values can be physically implemented is the pertinence or not of the point (i.e., $L_{c}{ }^{*}, C_{c}{ }^{*}$ ) to the region enclosed by the polygon. It is possible to refine the convergence region by calculating more points, meaning further values/combinations of $c$ and $d$ (for instance those corresponding to a combination of a extreme and an intermediate value).

As a representative example of a CSRR-loaded line model, we have chosen $L^{*}=4.86 \mathrm{nH}$ and $C^{*}=1.88 \mathrm{pF}$. The convergence region for the subspace $L_{c}-C_{c}$ calculated following the procedure explained above is depicted in Fig. 3(a). Obviously, decreasing the maximum allowable value of $c_{\max }$ and $d_{\max }$ has the effect of reducing the area of the polygon, as Fig. 3(b) illustrates. However, $c_{\max }=d_{\max }=0.4$ $\mathrm{mm}$ is a reasonable value that represents a tradeoff between accuracy (related to the frequency responses of the fine and coarse models) and size of the convergence region.
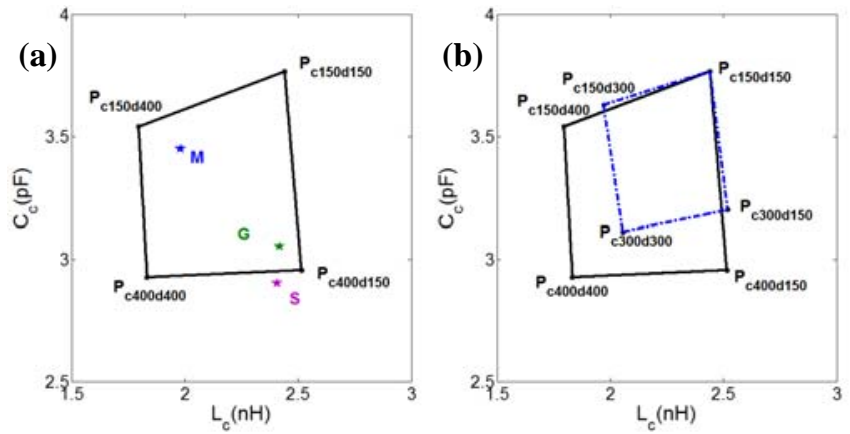

Fig.3. (a) Convergence region for a CSRR-loaded line model with $L^{*}=4.86$ $\mathrm{nH}$ and $C^{*}=1.88 \mathrm{pF}$, defined by four points in the $L_{c}-C_{c}$ subspace, and considering $c_{\min }=d_{\min }=0.15 \mathrm{~mm}$ and $c_{\max }=d_{\max }=0.4 \mathrm{~mm}$. (b) Various regions defined with different constraints for the same values of $L^{*}$ and $C^{*}$. The thickness of the substrate is $h=1.27 \mathrm{~mm}$.

The other case under study, i.e., CSRR-gap-loaded metalines, presents a coarse model which depends on 5 variables, those of the line without gap plus $C_{g}$ (Fig. 1a). The additional geometrical variable is simply the gap space, $s$. The convergence region can be obtained following a similar procedure, but now the target response is given by the electrical parameters that characterize the microstrip line with gap $\left(L^{*}, C^{*}\right.$ and $\left.C_{g}^{*}\right)$. The pre-optimization ASM algorithm is thus more complex since it involves three variables, being the line length, $l$, width, $W$, and gap space, $s$, the unknown dimension parameters to be calculated. As in the previous case, the initial layout for the first vertex is found analytically, as it is detailed in Appendix B. Once the geometry of the line with gap is estimated, the electrical parameters are extracted according to the procedure given in Appendix A, the Broyden matrix is initiated, and the process is iterated until convergence is achieved. The result is a set of circuit model element values corresponding to the target elements of $L^{*}, C^{*}$ and $C_{g}{ }^{*}$, and $L_{c}$ and $C_{c}$ values that depend on the considered CSRR geometry.

One differential aspect of CSRR-gap-loaded lines, as compared to CSRR-loaded lines, is the number of necessary points in the $L_{c}-C_{c}$ subspace to accurately determine the convergence region. It has been found that four points do not suffice in this case. Hence, the pre-optimization ASM algorithm has been applied eight times: four of them by considering the CSRR geometries with extreme values of $c$ and $d$; the other four cases by considering the following combinations: $c_{\text {min }}-d_{\text {mean }}, c_{\text {max }}-d_{\text {mean }}, c_{\text {mean }}-d_{\text {max }}$, and $c_{\text {mean }}-d_{\text {min }}$, where $d_{\text {mean }}=\left(d_{\text {min }}+d_{\text {max }}\right) / 2$ and $c_{\text {mean }}=\left(c_{\text {min }}+c_{\text {max }}\right) / 2$.

In some occasions, where a large gap capacitance is required, it is convenient to use a gap with T-shaped geometry (Fig. 4). In this case, in order to preserve the number of variables in the validation space, the gap distance, $s$, is set to a small value (this helps to increase the series capacitance of the gap), and the gap width, $W_{g}$, is considered as the geometry variable associated to the presence of the gap. Thus, the gap distance is set to $s=0.2 \mathrm{~mm}$ (notice that $l_{g}$-see Fig. 4 - is also 
set to $l_{g}=0.2 \mathrm{~mm}$ to avoid an excess of variables). However, the pre-optimizer and core ASM algorithms do not experience any change by considering T-shaped gaps.

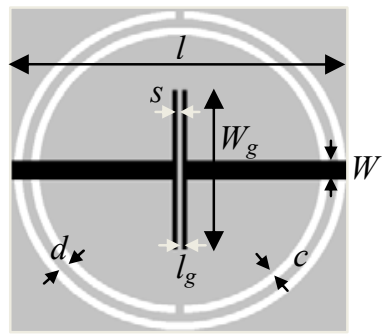

Fig. 4. CSRR-based microstrip line with T-shaped gap.

Figure 5 depicts the convergence region in the $L_{c}-C_{c}$ subspace that results by considering $L^{*}=9.45 \mathrm{nH}$ and $C^{*}=$ $17.93 \mathrm{pF}$ and $C_{g}{ }^{*}=1.01 \mathrm{pF}$ for a CSRR-gap-loaded line with T-shaped gap. In view of this figure, it is clear that four points are not enough to determine the convergence region (notice that the $L_{c}-C_{c}$ points corresponding to the CSRR geometries given by $c_{\text {min }}-d_{\text {mean }}, c_{\text {max }}-d_{\text {mean }}, c_{\text {mean }}-d_{\text {max }}$, and $c_{\text {mean }}-d_{\text {min }}$ are significantly misaligned with the lines forming the polygon resulting by considering the four cases with extreme values of $c$ and $d$ ).

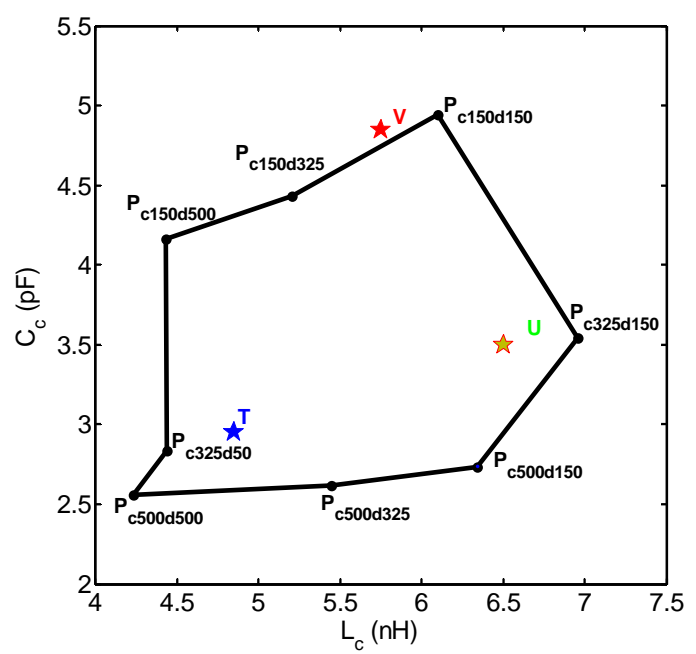

Fig. 5. Convergence region for a CSRR-gap-loaded line model with $L^{*}=9.45$ $\mathrm{nH}, C^{*}=17.93 \mathrm{pF}$, and $C_{g}{ }^{*}=1.01 \mathrm{pF}$, defined by eight points in the $L_{c}-C_{c}$ subspace, and considering $c_{\min }=d_{\min }=0.15 \mathrm{~mm}$ and $c_{\max }=d_{\max }=0.5 \mathrm{~mm}$. The thickness of the substrate is $h=0.635 \mathrm{~mm}$.

\section{B. Determination of the Initial Layout}

Let us consider the synthesis of a given set of circuit parameters of the circuit model (target coarse model solution), for a CSRR-loaded metaline, and let us assume that the previous analysis reveals that such target parameters in the coarse model space have an implementable fine model solution. The next step is the determination of the initial layout, unless $L_{c}{ }^{*}$ and $C_{c}{ }^{*}$ coincide with any of the vertices of the converging polygon in the $L_{c}-C_{c}$ subspace (in this case the layout is already known and hence no further optimization is necessary). From the previous analysis, it is expected that the dimensions of the CSRR after ASM optimization depend on the position of the $L_{c}{ }^{*}-C_{c}{ }^{*}$ point in the convergence region. Namely, if the $L_{c}{ }^{*}-C_{c}{ }^{*}$ point is close to a vertex, it is expected that $c$ and $d$ are similar to the values corresponding to that vertex.

In this paper, a new procedure to determine the initial layout, necessary to start-up the core ASM algorithm, is proposed. First, the determination of the initial layout for CSRR-loaded lines is considered. The aim is to express any of the geometrical variables $\left(c, d, l\right.$ or $W$ ) as a function of $L_{c}$ and $C_{c}$. To obtain the initial value of each geometrical dimension involved in the optimization process, we will assume it has a linear dependence with $L_{c}$ and $C_{c}$. For instance, the initial value of $c$ (for the other variables we will use identical expressions) will be estimated according to:

$$
c=f\left(L_{c}, C_{c}\right)=\left(A+B L_{c}\right)\left(C+D C_{c}\right)
$$

The previous expression can be alternatively written as:

$$
c=f\left(L_{c}, C_{c}\right)=a_{0}+a_{1} L_{c}+a_{2} C_{c}+a_{3} L_{c} C_{c}
$$

where the constants $a_{i}$ determine the functional dependence of the initial value of $c$ with $L_{c}$ and $C_{c}$. To determine the constants $a_{i}$, four conditions are needed. Let us consider the following error function:

$$
f_{\text {error }}=\sum_{j=1}^{N_{v}}\left(c_{j}-f\left(L_{c_{j}}, C_{c_{j}}\right)\right)^{2}
$$

where the subscript $j$ is used to differentiate between the different vertices, and hence $c_{j}$ is the value of $c$ in the vertex $j$, and $L_{c j}, C_{c j}$ the corresponding values of $L_{c}$ and $C_{c}$ for that vertex. We have considered a number of vertices equal to 4 (corresponding to CSRR-loaded lines), but the formulation can be generalized to a higher number of vertices. Expression (12) can then be written as:

$$
\begin{aligned}
f_{\text {error }}= & \sum_{j=1}^{4} c_{j}^{2}-2 \sum_{j=1}^{4} c_{j} \cdot\left(a_{0}+a_{1} L_{c_{j}}+a_{2} C_{c_{j}}+a_{3} L_{c_{j}} C_{c_{j}}\right)+ \\
& +\sum_{j=1}^{4}\left(a_{0}+a_{1} L_{c_{j}}+a_{2} C_{c_{j}}+a_{3} L_{c_{j}} C_{c_{j}}\right)^{2}
\end{aligned}
$$

To find the values of the constants $a_{i}$, we obtain the partial derivatives of the previous error function with regard to $a_{i}$, and force them to be equal to zero [40]:

$$
\begin{gathered}
\frac{\partial f_{\text {error }}}{\partial a_{i}}=-2 \sum_{j=1}^{4} c_{j} \frac{\partial f\left(L_{c_{j}}, C_{c_{j}}\right)}{\partial a_{i}}+ \\
+2 \sum_{j=1}^{4}\left[f\left(L_{c_{j}}, C_{c_{j}}\right) \frac{\partial f\left(L_{c_{j}}, C_{c_{j}}\right)}{\partial a_{i}}\right]=0
\end{gathered}
$$

for $i=1,2,3,4$. Following this least-squares approach, four independent equations for the constants $a_{i}$ are obtained. Such equations can be written in matrix form as follows:

$$
\left(\begin{array}{cccc}
4 & \sum_{j=1}^{4} L_{c_{j}} & \sum_{j=1}^{4} C_{c_{j}} & \sum_{j=1}^{4} L_{c_{j}} C_{c_{j}} \\
\sum_{j=1}^{4} L_{c_{j}} & \sum_{j=1}^{4} L_{c_{j}}^{2} & \sum_{j=1}^{4} L_{c_{j}} C_{c_{j}} & \sum_{j=1}^{4} L_{c_{j}}^{2} C_{c_{j}} \\
\sum_{j=1}^{4} C_{c_{j}} & \sum_{j=1}^{4} L_{c_{j}} C_{c_{j}} & \sum_{j=1}^{4} C_{c_{j}}^{2} & \sum_{j=1}^{4} C_{c_{j}}^{2} L_{c_{j}} \\
\sum_{j=1}^{4} L_{c_{j}} C_{c_{j}} & \sum_{j=1}^{4} L_{c_{j}}^{2} C_{c_{j}} & \sum_{j=1}^{4} C_{c_{j}}^{2} L_{c_{j}} & \sum_{j=1}^{4} C_{c_{j}}^{2} L_{c_{j}}^{2}
\end{array}\right)\left(\begin{array}{l}
a_{o} \\
a_{1} \\
a_{2} \\
a_{3}
\end{array}\right)=
$$




$$
\left(\begin{array}{c}
\sum_{j=1}^{4} c_{j} \\
\sum_{j=1}^{4} c_{j} L_{c_{j}} \\
\sum_{j=1}^{4} c_{j} C_{c_{j}} \\
\sum_{j=1}^{4} c_{j} L_{c_{j}} C_{c_{j}}
\end{array}\right)
$$

Once the constants $a_{i}$ are obtained (solving the previous equations), the initial value of $c$ is inferred from (11). The process is repeated for $d, W$ and $l$, and the initial geometry necessary for the initiation of the ASM algorithm is thus obtained.

For the CSRR-gap-loaded lines, a similar approach is followed, although the number of vertices is eight, rather than four. As compared to the algorithm reported in [34],[35], the initial geometry derived from the procedure explained in this sub-section is very close to the final geometry, and this is fundamental to reduce the number of steps towards ASM convergence, as it will be shown later. As compared to previous works by the authors, the main relevant contributions of the present paper are the determination of the initial layout from the least squares approach, and the preoptimizer ASM algorithm to determine the convergence region in the $L_{c}-C_{c}$ space. These are fundamental aspects to make the synthesis approach practical and competitive.

\section{The core ASM algorithm}

Once the initial geometry is calculated, the response of the fine model is obtained through EM analysis, and the circuit parameters are extracted, from which the error function (6) can be obtained. To initiate the Broyden matrix, we slightly perturb each geometrical variable from the value corresponding to the initial layout, and we obtain the circuit parameters resulting from each geometry variation. The relative changes can be expressed in a matrix form as follows:

$$
\left(\begin{array}{cccc}
\frac{\delta L}{\delta W} & \frac{\delta L}{\delta l} & \frac{\delta L}{\delta c} & \frac{\delta L}{\delta d} \\
\frac{\delta C}{\delta W} & \frac{\delta C}{\delta l} & \frac{\delta C}{\delta c} & \frac{\delta C}{\delta d} \\
\frac{\delta L_{c}}{\delta W} & \frac{\delta L_{c}}{\delta l} & \frac{\delta L_{c}}{\delta c} & \frac{\delta L_{c}}{\delta d} \\
\frac{\delta C_{c}}{\delta W} & \frac{\delta C_{c}}{\delta l} & \frac{\delta C_{c}}{\delta c} & \frac{\delta C_{c}}{\delta d}
\end{array}\right)
$$

which corresponds to the initial Broyden matrix values. Once the Broyden matrix is known, the geometry of the following iteration can be derived from (7), and the process is iterated until convergence is obtained. To avoid that the variables in the fine model space exceed the limits of the implementable range of values with the available technology, geometrical constraints and a shrinking factor $\delta$ are introduced. As it can be seen in Fig. 6, the vector $\boldsymbol{x}_{\boldsymbol{f}}$ is tested, and if some of the geometrical variables exceed the geometrical constraints, the algorithm uses the shrinking factor in order to obtain a new vector $\boldsymbol{x}_{\boldsymbol{f}}$ inside the limits.
The iterative algorithm is controlled by the MATLAB ${ }^{(\mathrm{R})}$ commercial software [41], which is the core tool of the implemented ASM algorithm. The three main building blocks of the MATLAB program are the initial geometry calculator (that previously determines the convergence regions in the $L_{c^{-}}$$C_{c}$ subspace as explained before), the EM solver, and the parameter extractor. For the EM simulation, we have considered two alternative options: the Agilent Momentum simulator (incorporated in the Agilent ADS commercial software) [42], and Ansoft Designer [43]. The EM solver creates the geometry of the structure, which is then exported to either Agilent Momentum or Ansoft Designer to carry out the EM simulation. The results of this simulation (the Sparameters) are then imported back to MATLAB and processed by the parameter extraction module, which allows us to extract the circuit parameters. These values are compared with the target parameters, and the error function is obtained according to:

$$
\left\|f_{\text {norm }}\right\|=\sqrt{\left(1-\frac{L}{L^{*}}\right)^{2}+\left(1-\frac{C}{C^{*}}\right)^{2}+\left(1-\frac{L_{c}}{L_{c}^{*}}\right)^{2}+\left(1-\frac{C_{c}}{C_{c}^{*}}\right)^{2}}
$$

From this, the next geometry of the iterative process is inferred following the update procedure described before. The flow diagram of the complete ASM algorithm (including constraints to avoid unwanted solutions) is depicted in Fig. 6.

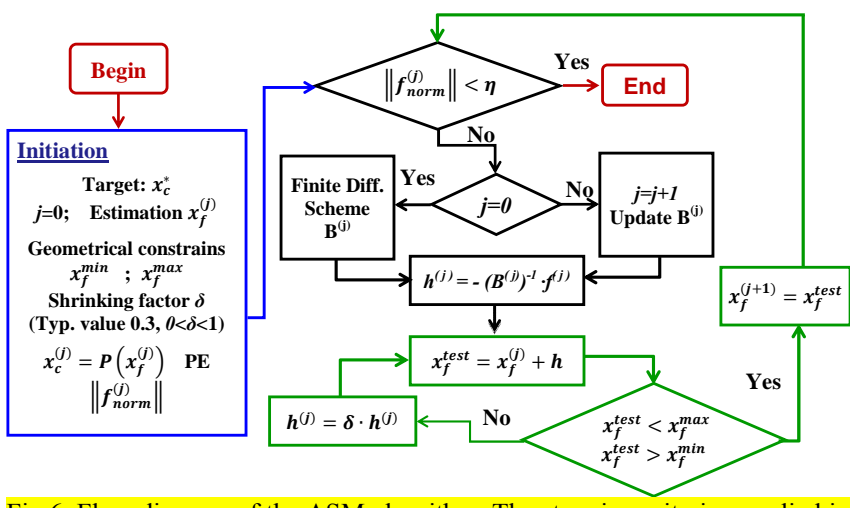

Fig.6. Flow diagram of the ASM algorithm. The stopping criterion applied in this work is $\left\|f_{\text {norm }}{ }^{(j)}\right\|<\eta<<1$

\section{Effects of the new ASM algorithm on convergence speed}

The main novelty of the proposed ASM algorithm is the calculation of a very good initial geometry. First, we have applied the new ASM algorithm to the CSRR-loaded metalines whose optimal coarse parameters are detailed in Table I, using Ansoft Designer as the EM-solver. In Fig. 3(a) the location of these three different cases with respect to the shared convergence region (they have the same $L^{*}$ and $C^{*}$ values) is shown. Notice that in the case that the point is out of the convergence region but close to it, we let the algorithm to continue and change the limiting constrains of $c$ and $d$.

The evolution of the error function with the iteration number is plotted in Fig. 7, and compared to that of our previous ASM algorithm [34], [35]. Convergence is much faster with the new ASM approach since the initial layout is closer to the final solution. It is noticeable that there are points, such as $\mathrm{S}$, that do not converge to a final solution with 
our previous ASM algorithm, whereas now convergence is obtained with the new approach. The number of iterations that needs the ASM algorithm to converge to the final layout dimensions and its corresponding evaluated error are summarized in Table II, for the three different examples considered ( $\eta$ has been set to 0.01 ).

TABLE I. OPTIMAL COARSE SOLUTION

\begin{tabular}{|c|c|c|c|c|}
\cline { 2 - 5 } \multicolumn{1}{c|}{} & \multicolumn{4}{c|}{$\boldsymbol{x}_{c}{ }^{*}$} \\
\hline S & 4.860 & 1.880 & 2.407 & 2.902 \\
\hline G & 4.860 & 1.880 & 2.417 & 3.053 \\
\hline M & 4.860 & 1.880 & 1.980 & 3.450 \\
\hline
\end{tabular}

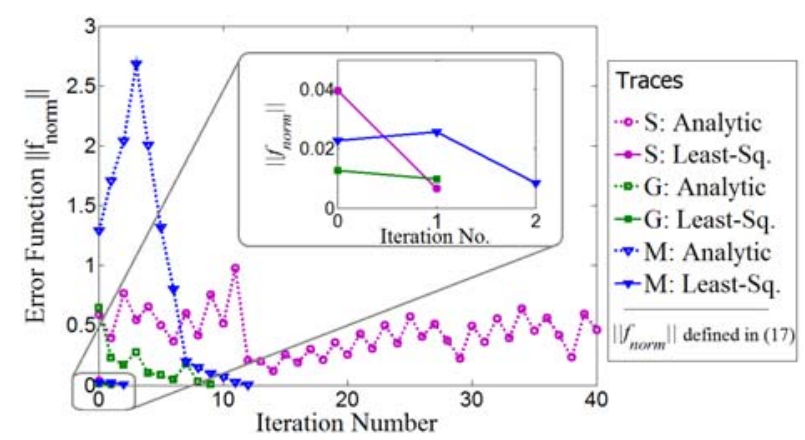

Fig. 7. Evolution of the error function of the ASM algorithm for the points of Table I. Dashed lines correspond to the evolution of the error function by considering the initial layout resulting from analytical expresssions [34],[35]; solid lines give the error function for the initial layout calculated from the least-squares approach proposed in this paper. M (blue), G (green) and S (pink) points.
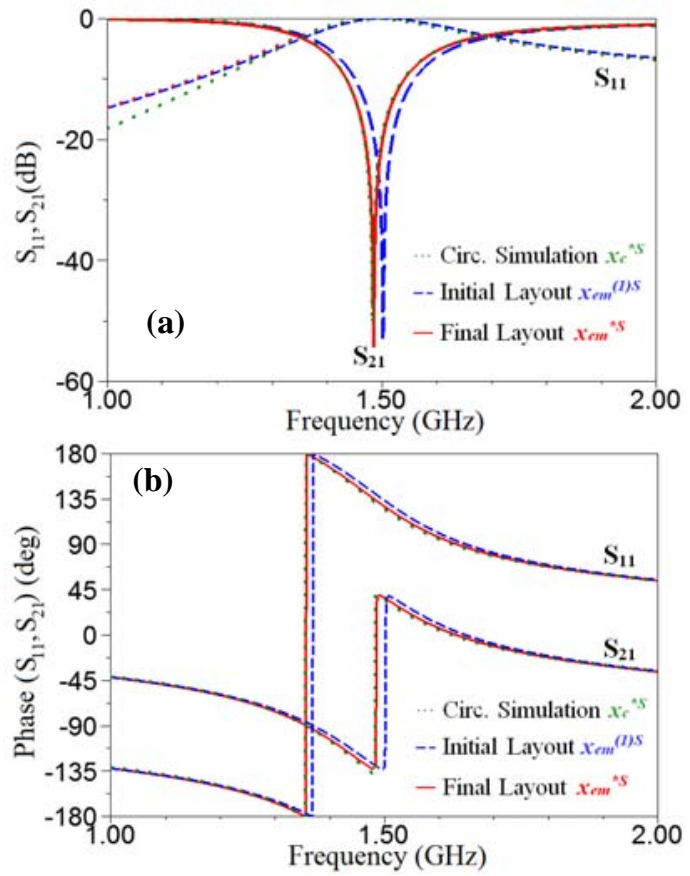

Fig. 8. Magnitude (a) and phase (b) of the scattering parameters $S_{21}$ and $S_{11}$ at initial solution $\boldsymbol{x}_{e m}{ }^{(1)}, \boldsymbol{x}_{e m}{ }^{*}$, and circuital simulation of the $\boldsymbol{x}_{c}{ }^{*}$ for point $\mathrm{S}$.

The final layout obtained for the first example (S) is slightly out of the lower boundary $(c=0.41 \mathrm{~mm})$, as it was expected since the target $\mathrm{S}$ was placed out of the convergence region (see Fig. 3a). However, we have let the algorithm to continue towards convergence by relaxing the limiting values of $c$ and $d$. Thus, the fact that the target is out of the convergence region does not necessarily mean that convergence is not possible, but that the resulting geometric values might be beyond or below the considering limits of $c$ and $d$. For the $\mathrm{S}$ point, the close proximity to the line of the polygon corresponding to $c=0.4 \mathrm{~mm}$ (Fig. 3) explains that the final value of $c$ is $0.41 \mathrm{~mm}$. The agreement between the frequency response obtained by EM simulation of the final layout (obtained after a single iteration) and the circuit simulation of the target parameters is excellent, as it can be seen in Fig. 8. This is reasonable since the value of $c$ is not far from the considering limiting value that guarantees that the CSRRs are accurately described by the models depicted in Fig. 1.

Figure 9 depicts the comparison of the frequency responses corresponding to the point $\mathrm{G}$, where, again, very good agreement between circuit simulation (target parameters) and EM simulation of the final layout results.

TABLE II. FINAL LAYOUT

\begin{tabular}{|c|c|c|c|c|c|c|}
\hline \multirow{2}{*}{ Case } & \multicolumn{4}{|c|}{$x_{e m}{ }^{*}$} & \multirow{2}{*}{$\begin{array}{c}\text { Iter. } \\
\text { no. }\end{array}$} & \multirow{2}{*}{$\begin{array}{l}\text { Error } \\
\left\|f_{\text {norm }}\right\|\end{array}$} \\
\hline & $l^{\mathrm{a}}$ & $W^{\text {a }}$ & $c^{\mathrm{a}}$ & $d^{\mathrm{a}}$ & & \\
\hline Analytic $S$ & 8.34 & 2.21 & 0.47 & 0.22 & 40 & 0.1192 \\
\hline Least-squares $S$ & 8.41 & 2.28 & 0.41 & 0.16 & 1 & 0.0066 \\
\hline Analytic $G$ & 8.44 & 2.23 & 0.35 & 0.17 & 9 & 0.0057 \\
\hline Least-squares $G$ & 8.47 & 2.22 & 0.36 & 0.17 & 1 & 0.0098 \\
\hline Analytic $M$ & 8.56 & 2.21 & 0.18 & 0.32 & 12 & 0.0038 \\
\hline Least-squares $M$ & 8.59 & 2.20 & 0.18 & 0.33 & 2 & 0.0084 \\
\hline
\end{tabular}
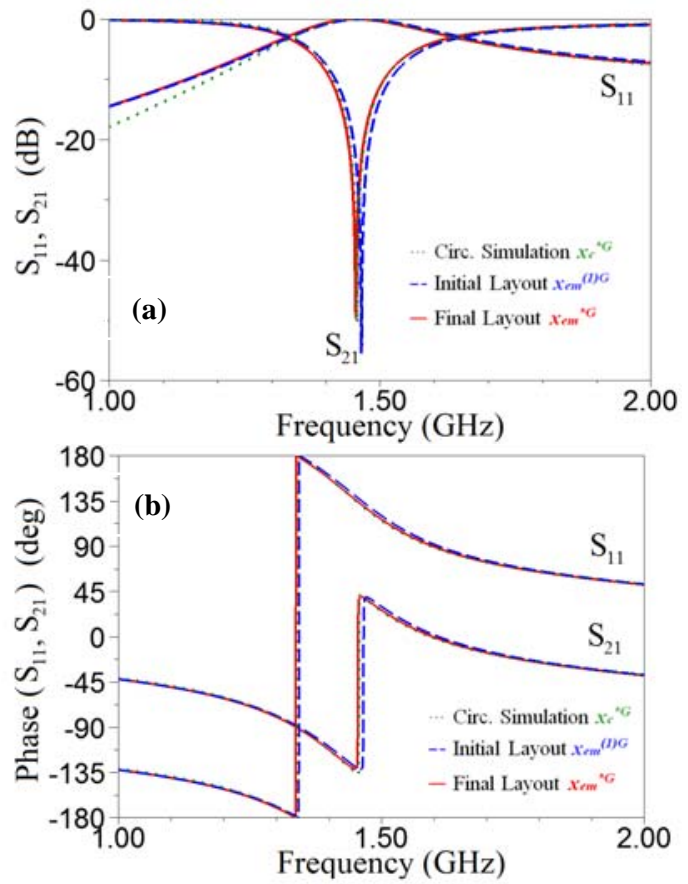

Fig. 9. Magnitude (a) and phase (b) of the scattering parameters $S_{21}$ and $S_{11}$ at initial solution $\boldsymbol{x}_{\boldsymbol{e m}}{ }^{(\mathbf{1})}, \boldsymbol{x}_{\boldsymbol{e m}}{ }^{*}$, and circuital simulation of $\boldsymbol{x}_{c}{ }^{*}$ for point $\mathrm{G}$. 
TABLE III. OPTIMAL COARSE SOLUTION

\begin{tabular}{|c|c|c|c|c|c|}
\cline { 2 - 6 } \multicolumn{1}{c|}{} & \multicolumn{5}{c|}{$\boldsymbol{x}_{\boldsymbol{c}}{ }^{*}$} \\
\hline $\mathbf{T}$ & 9.45 & 17.93 & 1.01 & 4.85 & 2.95 \\
\hline $\mathbf{U}$ & 9.45 & 17.93 & 1.01 & 6.50 & 3.50 \\
\hline $\mathrm{V}$ & 9.45 & 17.93 & 1.01 & 5.75 & 4.85 \\
\hline
\end{tabular}

We have also obtained the error function with the iteration number for CSRR-gap (T-shaped) loaded lines with the targets indicated in Fig. 5 and given in table III (the target $\mathrm{T}$ corresponds to a dual-band impedance inverter, which is the artificial line used in one of the examples discussed in section IV). The resulting geometrical values and number of required iterations are given in Table IV (in this case $\eta$ has been set to 0.04 , still providing very accurate results). Figure 10 depicts the frequency response for the initial and final layout, as well as the circuit simulation of the target parameters for the $T$ case (the considered electromagnetic solver for these CSRRgap-loaded lines is the Agilent Momentum).
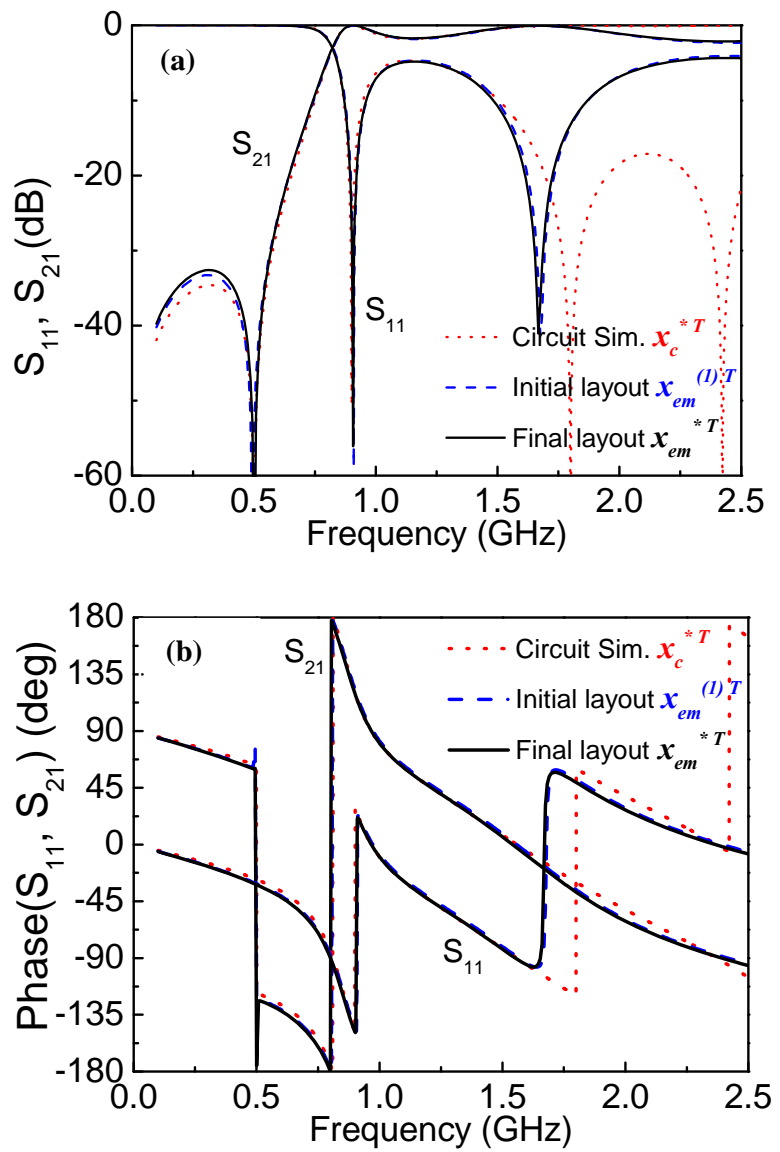

Fig. 10. Magnitude (a) and phase (b) of the scattering parameters $S_{21}$ and $S_{11}$ at initial solution $\boldsymbol{x}_{e m}{ }^{(1)}, \boldsymbol{x}_{e m}{ }^{*}$, and circuital simulation of $\boldsymbol{x}_{c}{ }^{*}$ for point $\mathrm{T}$. Deviations between the circuit simulation and the electromagnetic response of the final layout are due to the fect that the circuit model is not valid at high frequencies.

It should be noted that the initial layout (inferred from the pre-optimization algorithm) is already very close to the final solution. However, it can be observed a progressive mismatch between the electromagnetic response of the final layout and the circuit simulation as frequency increases due to the inaccuracy of the circuit model at high frequencies. We do not provide the convergence curves that lead to the number of iterations and error functions indicated in Table IV, since we cannot compare such curves with those curves that result by using the previous version of the ASM algorthm (i.e., that reported in [39]). The reason is that our old ASM algorithm was not adapted for T-shaped gaps. Nevertheless, convergence speed is good (few iterations suffice to obtain a small error function), although not so fast, as compared to the structures without gap (Fig. 7).

TABLE IV. FINAL LAYOUT

\begin{tabular}{|c|c|c|c|c|c|c|c|}
\hline \multirow{2}{*}{ Case } & \multicolumn{5}{|c|}{$\boldsymbol{x}_{e m}{ }^{*}$} & \multirow{2}{*}{$\begin{array}{c}\text { Iter. } \\
\text { no. }\end{array}$} & \multirow{2}{*}{$\begin{array}{l}\text { Error } \\
\left\|f_{\text {norm }}\right\|\end{array}$} \\
\hline & $l^{\text {a }}$ & $\boldsymbol{W}^{\text {a }}$ & $c^{\mathrm{a}}$ & $d^{\mathrm{a}}$ & $\boldsymbol{W}_{g}^{\mathrm{a}}$ & & \\
\hline $\mathbf{T}$ & 14.2 & 0.87 & 0.34 & 0.40 & 7.13 & 6 & 0.0124 \\
\hline $\mathbf{U}$ & 15.7 & 0.68 & 0.33 & 0.16 & 6.61 & 13 & 0.0292 \\
\hline V & 17.1 & 0.56 & 0.13 & 0.21 & 6.07 & 13 & 0.0386 \\
\hline
\end{tabular}

\section{APPLICATION EXAMPLES}

\section{A. Stop-band filter}

A practical application of CSRR-loaded lines without gaps is the implementation of stop-band filters with controllable response (bandwidth and rejection level). Compact planar stop-band filters can be designed by cascading several CSRRloaded line unit cells. Let us illustrate this possibility through the design procedure and experimental verification of a three unit cell stop-band filter in microstrip technology.

The first step consists on the synthesis of the central unit cell, whose transmission zero frequency, $f_{z}$, is chosen to be the central frequency of the stop-band, i.e., $2.45 \mathrm{GHz}$. Since the other cells involved in the design will have similar target responses, and very close transmission zero frequencies (i.e. $2.36 \mathrm{GHz}$ and $2.53 \mathrm{GHz}$ ), the optimal coarse solutions for all the involved cells were forced to be placed in the same convergence region (i.e. with the same $L$ and $C$ values, as it is seen in Table V) in order to speed up the design process. By cascading the three cells with the circuit elements indicated in Table V, a satisfactory stop-band response at the circuit simulation level results. By using the ASM algorithm described in Section III, and considering the Rogers RO3010 substrate with thickness $h=1.27 \mathrm{~mm}$ and dielectric constant $\varepsilon_{r}=10.2$, the three layouts summarized in Table VI have been obtained.

TABLE V. Optimal CoArse Solutions Of Unit Cells

\begin{tabular}{|l|c|c|c|c|}
\hline \multicolumn{1}{c|}{} & \multicolumn{4}{c|}{$\boldsymbol{x}_{\boldsymbol{c}}{ }^{*}$} \\
\cline { 2 - 5 } & $\boldsymbol{L}[\mathbf{n H}]$ & $\boldsymbol{C}_{\mathbf{p} F]}$ & $\boldsymbol{L}_{\boldsymbol{c}}[\mathbf{n H}]$ & $\boldsymbol{C}_{\boldsymbol{c}}[\mathbf{p F}]$ \\
\hline $\mathbf{C}_{2.36}$ & 3.194 & 1.363 & 1.009 & 3.143 \\
\hline $\mathbf{C}_{2.45}$ & 3.194 & 1.363 & 0.941 & 3.120 \\
\hline $\mathbf{C}_{\mathbf{2 . 5 3}}$ & 3.194 & 1.363 & 0.882 & 3.125 \\
\hline
\end{tabular}


TABLE VI. SyNTHESISED LAYOUTS For THE DIFFERENT UNIT CELLS

\begin{tabular}{|c|c|c|c|c|c|c|}
\hline \multirow[t]{2}{*}{ Cell $\left(f_{z}\right)$} & \multicolumn{4}{|c|}{$\boldsymbol{x}_{e m}{ }^{*}$} & \multirow{2}{*}{$\begin{array}{l}\text { Iter. } \\
\text { no. }\end{array}$} & \multirow{2}{*}{$\begin{array}{l}\text { Error } \\
\left\|f_{\text {norm }}\right\|\end{array}$} \\
\hline & $l^{\text {a }}$ & $W^{\text {a }}$ & $c^{\mathrm{a}}$ & $d^{\text {a }}$ & & \\
\hline $2.36 \mathrm{GHz}$ & 5.93 & 2.16 & 0.28 & 0.25 & 3 & 0.0074 \\
\hline $2.45 \mathrm{GHz}$ & 5.91 & 2.23 & 0.34 & 0.27 & 1 & 0.0055 \\
\hline $2.53 \mathrm{GHz}$ & 5.93 & 2.25 & 0.34 & 0.33 & 5 & 0.0069 \\
\hline
\end{tabular}

However, it is expected that by directly cascading the three designed cells, coupling effects between the CSRRs appear (this has been verified through EM simulation, not shown). Therefore, it is necessary to insert transmission line sections between the synthesized unit cells, as depicted in Figs. 11 and 12. The length of the transmission line sections between adjacent CSRRs has been set to $3 / 4$ the CSRR radius (the width being identical to that of the microstrip on top of the CSRR, indicated in Table VI). With these transmission line lengths, coupling effects between adjacent CSRRs are not present, while the circuit response does not substantially change as compared to the case with direct connection between unit cells at the circuit level.

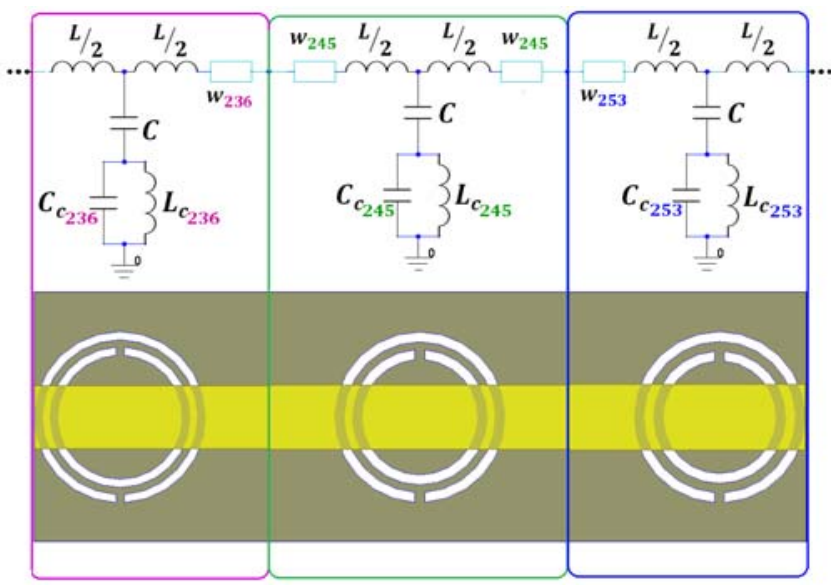

Fig. 11. Equivalent circuit and layout of the implementer stop-band filter. The small blue rectangles correspond to lines of physical length equal to $2.22 \mathrm{~mm}$ and width according to the value for each unit cell (see Table VI).

The completed manufactured stop-band filter can be seen in Figure 12, where tapers between the input/output $50 \Omega$ transmission lines (needed to perform the measurements) and the designed structure are included. It was fabricated by using the circuit board plotter LPKF ProMat S103.

The measured filter response, displayed in Fig. 13, is compared with the equivalent circuit and full-wave electromagnetic simulated responses. The measured rejection is better than $20 \mathrm{~dB}$ within a $345 \mathrm{MHz}$ frequency band. The agreement between circuit and electromagnetic simulation is very good, as expected on account of the small errors that appear in Table VI. The small discrepancies in measurement and the additional reflexion zero are attributed to fabrication related tolerances.

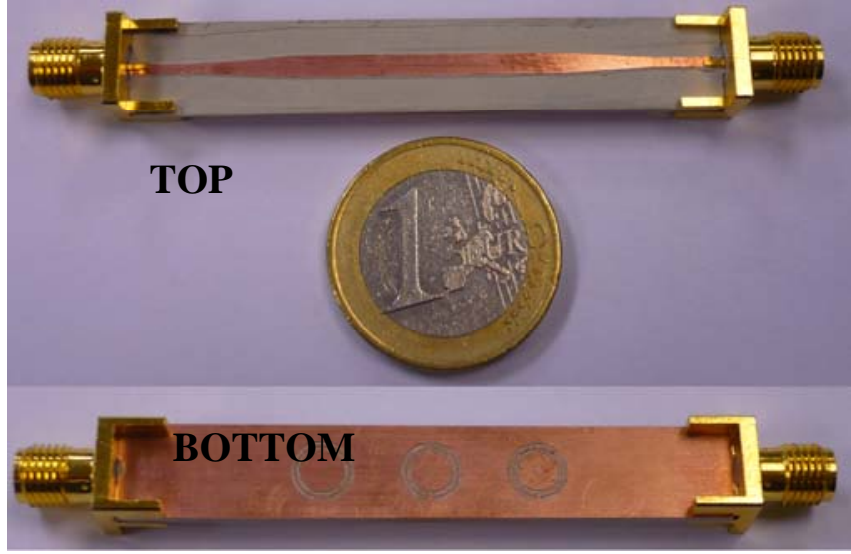

Fig. 12. Photograph of the fabricated CSRR-based stop-band filter.

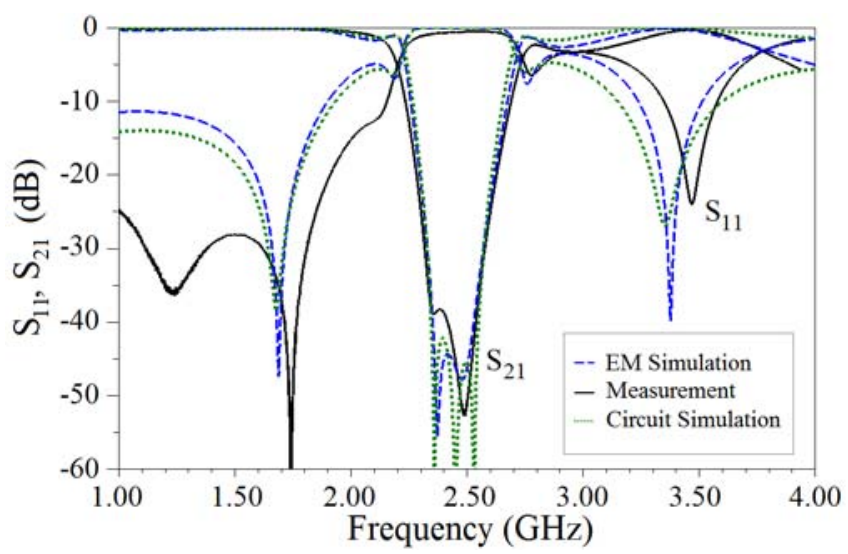

Fig. 13. Frequency response of the stop-band filter: the target response (i.e., that corresponding to the equivalent cirtuit simulation) is depicted in greendotted line, the full-wave EM simulation in blue-dashed line, and the measurements in black-solid lines.

\section{B. Dual band CSRR-based power divider}

Let us now consider the application of the proposed two-step ASM algorithm to the synthesis of a dual-band power splitter based on a dual-band impedance inverter implemented by means of the CSRR-gap-loaded line. To achieve the dualband functionality, the inverter must provide a phase shift of $-90^{\circ}$ and $+90^{\circ}$ at the operating frequencies, $f_{1}$ and $f_{2}$, respectively. We are thus exploiting the composite right/left handed behavior of the structure, with $f_{1}$ and $f_{2}$ located in the left handed and right handed regions, respectively (see [19]). The inverter impedance is set to $35.35 \Omega$ at both frequencies, in order to guarantee a good matching when the inverter output port is loaded with a pair of matched loads (in practice two $50 \Omega$ access lines) to implement the divider. Notice that these four conditions do not univocally determine the five circuit elements of the model of the CSRR-gap loaded line (Fig. 1a). However, we can set the transmission zero (see equation A.2 in appendix A) to a certain value, and the five parameters thus have a unique solution. Specifically, the design frequencies of the inverter have been set to $f_{1}=0.9$ $\mathrm{GHz}$ and $f_{2}=1.8 \mathrm{GHz}$ and the transmission zero to $f_{z}=0.5$ $\mathrm{GHz}$. 
With the previous specifications, the element values can be easily found from (A.2), (A.3) and the characteristic impedance of the symmetric T-network, given by:

$$
Z_{o}=\sqrt{Z_{s}(\omega)\left[Z_{s}(\omega)+2 Z_{p}(\omega)\right]}
$$

where $Z_{s}$ and $Z_{p}$ are the series and shunt impedance, respectively, of the T-circuit model of Fig. 1(a). The resulting element values are $L=12.5 \mathrm{nH}, C=24.9 \mathrm{pF}, C_{g}=1.25 \mathrm{pF}, L_{c}=$ $3.38 \mathrm{nH}$ and $C_{c}=5.10 \mathrm{pF}$. With the element values of $L, C$ and $C_{g}$, we have obtained the convergence region in the $L_{c}-C_{c}$ plane according to the method reported before, and the target values of $L_{c}$ and $C_{c}$ do not belong to such region. This means that it is not possible to implement the dual-band impedance inverter by merely considering the CSRR-gap-loaded line (some element values are too extreme). However, it is expected that by cascading transmission line sections at both sides of the CSRR-gap loaded line, the element values of the cell are relaxed, and a solution within the convergence region arises. Therefore, we have cascaded two identical $35.35 \Omega$ transmission line sections at both sides of our CSRR-gap loaded line. The width of these line sections is $1.127 \mathrm{~mm}$, corresponding to the indicated characteristic impedance in the considered substrate (the Rogers RO3010, with thickness $h=$ $0.635 \mathrm{~mm}$ and dielectric constant $\varepsilon_{r}=10.2$ ). Notice that by cascading such $35.35 \Omega$ lines, the electrical length at the operating frequencies is the sum of the electrical lengths of the lines and the CSRR-based cell. Thus, the phase condition that must satisfy the CSRR-gap loaded line can be expressed as $\phi_{1}=-90^{\circ}-2 \phi_{L}\left(f_{1}\right)$ and $\phi_{2}=+90^{\circ}-2 \phi_{L}\left(f_{2}\right)$, where $\phi_{1}$ and $\phi_{2}$ are the electrical lengths of the CSRR-based cell at the design frequencies $f_{1}$ and $f_{2}$, and $\phi_{L}$ is the phase introduced by the line at the indicated frequency.

We have made a parametric analysis consisting on obtaining the element values of the CSRR-based cell for different values of the length of the cascaded transmission line sections (and hence $\phi_{L}\left(f_{1}\right)$ and $\phi_{L}\left(f_{2}\right)$ ). The results are depicted in Fig. 14. It can be observed that for small values of $\phi_{L}\left(f_{l}\right), L$ and $C$ are too large for being implemented. Large $L$ means a small value of $W$, but this is not compatible with a large $C$ value. On the other hand, the values of $L_{c}$ and $C_{c}$ without cascaded line sections, i.e., $\phi_{L}\left(f_{l}\right)=0^{\circ}$, give extreme values of $d$ and $c$, that is, large value of $d$ and small value of $c$. However, by increasing $\phi_{L}\left(f_{l}\right)$ (or the length of the cascaded lines), the variation of the elements of the central CSRR-gap based cell goes in the correct direction for their implementation. Specifically, we have considered a pair of transmission line sections with $\phi_{L}\left(f_{1}\right)=15^{\circ}$, which means that the required electrical lengths for the CSRR-based cell at the operating frequencies are $\phi_{1}=-120^{\circ}$ and $\phi_{2}=+30^{\circ}$. The element values corresponding to these phases are $L=9.45 \mathrm{nH}$ and $C=17.9$ $\mathrm{pF}, C_{g}=1.01 \mathrm{pF}, L_{c}=4.85 \mathrm{nH}$ and $C_{c}=2.95 \mathrm{pF}$, and these values lead us to an implementable layout. The reason is that this phase shift gives the minimum value of $L$ (see Fig. 14) and a reasonable small value of $C$, with $L_{c}$ not so small and $C_{c}$ no so large. Notice that $C_{g}$ does not experience significant variations with $\phi_{L}\left(f_{1}\right)$.

We have applied the proposed two-step ASM algorithm to the previous element values, and we have synthesized the layout of the CSRR-gap-loaded line, considering a T-shaped geometry for the gap. The geometrical parameters of the synthesized structure are (in reference to Fig. 4) $l=14.42 \mathrm{~mm}$, $W=0.87 \mathrm{~mm}, c=0.34 \mathrm{~mm}, d=0.40 \mathrm{~mm}, W_{g}=7.13 \mathrm{~mm}$, and convergence (with an error of 0.012) has been obtained after 6 iterations.

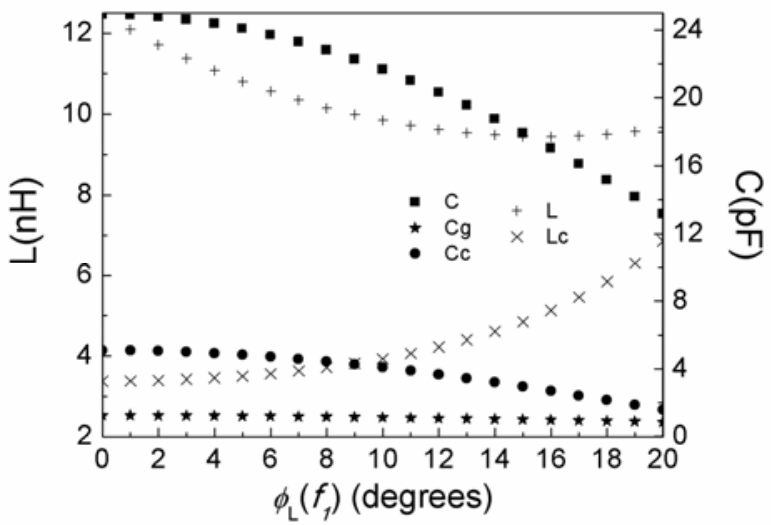

Fig. 14. Dependence of the element values of the CSRR-gap loaded line with the phase of each cascaded transmission line section at $f_{l}$.

The comparison of the electrical length and characteristic impedance inferred from EM simulation of the synthesized impedance inverter (the CSRR-based cell plus the cascaded $35.35 \Omega$ transmission line sections) and the ones inferred from circuit simulation are shown in Fig. 15. The agreement is excellent in the left handed region, where the model describes the structure to a very good approximation, and progressively degrades as frequency increases, as it is well known and expected. Nevertheless, the phase shift and the characteristic impedance at $f_{2}$ are reasonably close to the nominal values, and hence we do expect that the functionality of the power divider at $f_{2}$ is preserved.

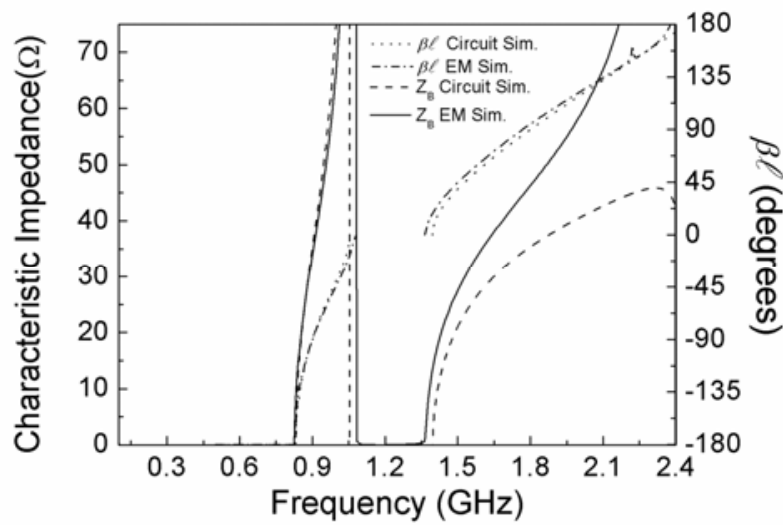

Fig. 15. Comparison between the electromagnetic and circuit simulation corresponding to the characteristic impedance and electrical length of the designed dual-band impedance inverter.

We have cascaded two output $50 \Omega$ access lines for connector soldering, and the structure has been fabricated by means of a photo/mask etching process (Fig. 16). Fig. 17 shows the frequency response of the divider, where it can be appreciated that optimum matching occurs at $f_{l}$ and slightly below $f_{2}$, for the reasons explained. Nevertheless, the functionality of the power divider covers both design frequencies. The discrepancy between the measured response and the target is not due to a failure of the ASM algorithm, 
but to the fact that the circuit model of the CSRR-gap loaded line does not accurately describe the structure at high frequencies, including part of the right handed band. A more accurate model increases the complexity of the ASM algorithm.

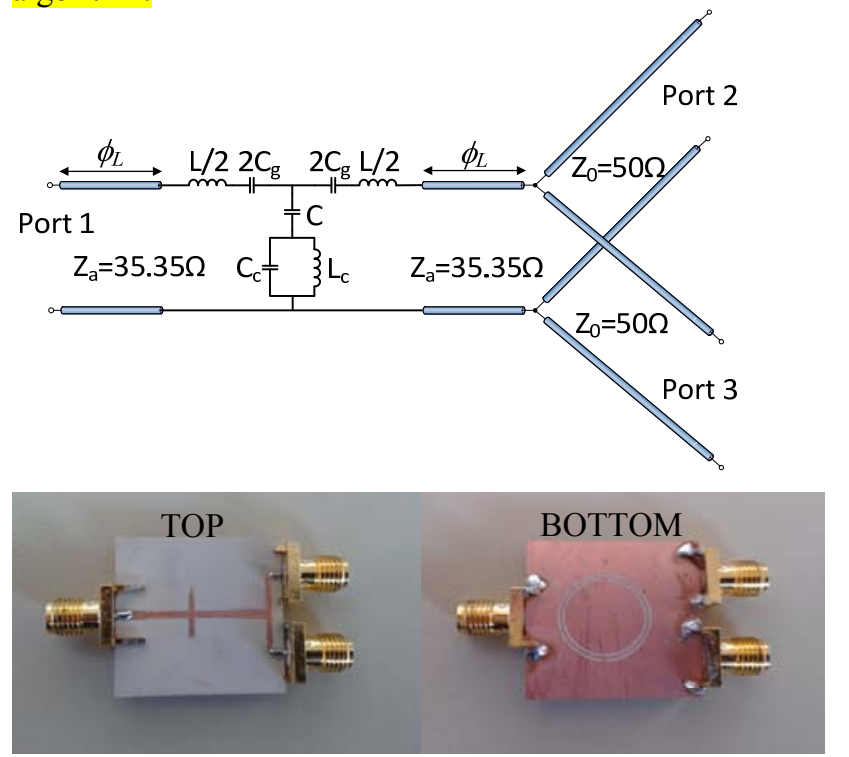

Fig. 16. Schematic and photograph of the fabricated dual-band power divider.

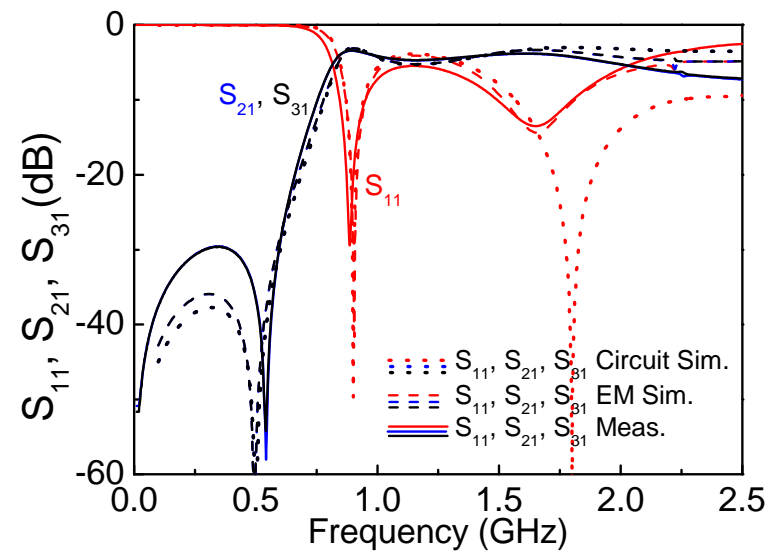

Fig. 17. Frequency response (circuit and electromagnetic simulation and measurement) of the designed and fabricated dual-band power divider.

\section{CONCLUSIONS}

In conclusion, a new tool for the automated synthesis of CSRR-based transmission lines, based on aggressive space mapping (ASM), has been proposed and developed. As compared to previous reported tools, the proposed algorithm is able to provide the convergence region in the coarse space; namely, it is able to predict whether a given set of element values of the equivalent circuit model of the unit cell is physically implementable or not. Moreover, the proposed tool is a two-step ASM algorithm, where the first (and fast) ASM optimizer is used not only to obtain the convergence region, but also to provide the necessary inputs (geometrical variables) for the determination of the initial layout from a novel developed least-squares approach. Thus, the initial layout is very close to the target, and the convergence time of the core (second step) ASM algorithm is dramatically decreased as compared to previous proposals (in some cases, two iterations have been enough to achieve convergence).

To illustrate the potential of the new two-step ASM algorithm to the synthesis of CSRR based devices, the tool has been used for the automated synthesis of a stop band filter and a dual-band power divider. In the former case, the filter is achieved by cascading three unit cells. In order to avoid coupling effects, that the equivalent circuit does not predict, small transmission line sections between adjacent cells have been added to the structure. Good agreement between the circuit simulation and the electromagnetic simulation with the layout of the CSRR-based cells obtained with the proposed algorithm has been achieved. The measured results are also in good agreement with the circuit and electromagnetic simulations, hence validating the designing process in the region of interest. For the dual-band power divider, the preoptimizer ASM has revealed that the target element values (inferred from the considered specifications) are not physically implementable. However, it has been found that by cascading two transmission line sections to the input and output ports, the element values of the unit cell are not so extreme, resulting in an implementable CSRR-gap loaded unit cell that has been synthesized by means of the proposed tool. The power divider has been fabricated and has been demonstrated to exhibit its functionality at the design frequencies.

The considered electromagnetic solvers of the new proposed ASM tool have been the Agilent Momentum and the Ansoft Designer commercial software packages (i.e., two versions of the two-step ASM algorithm have been developed). The tool can be extended to other available electromagnetic solvers. The results of this work open the path to the integration of this new ASM algorithm in such commercial tools for the fast and efficient optimization of CSRR-based circuits.

\section{APPENDIX A \\ PARAMETER EXTRACTION}

The parameters of the circuit of Fig. 1(a) can be extracted from the EM simulation of the CSRR-loaded line according to the following procedure. First, the reflection coefficient, $S_{11}$, is represented in the Smith Chart. At the intercept of $S_{11}$ with the unit resistance circle, the shunt branch opens and hence we can determine the resonance frequency of the CSRR

$$
f_{o}=\frac{1}{2 \pi \sqrt{L_{c} C_{c}}}
$$

as well as the value of the line inductance $L$ (from the simulated series reactance at $f_{o}$ ). To univocally determine the three circuit elements of the shunt branch we need two additional conditions, apart from expression (A1). One of them is the transmission zero frequency, $f_{z}$, which can be easily determined from the representation of the magnitude of $S_{21}$ with frequency. The transmission zero obeys the expression:

$$
f_{z}=\frac{1}{2 \pi \sqrt{L_{c}\left(C+C_{c}\right)}}
$$

The third required condition can be derived from the following expression providing the electrical length of the structure: 


$$
\cos (\phi)=1+\frac{Z_{s}(\omega)}{Z_{p}(\omega)}
$$

where $Z_{s}(\omega)$ and $Z_{p}(\omega)$ are the series and shunt impedance of the T-circuit model. Forcing $\phi=-\pi / 2$, it follows

$$
Z_{s}\left(\omega_{-\pi / 2}\right)=-Z_{p}\left(\omega_{-\pi / 2}\right)
$$

where $\omega_{-\pi / 2}$ is the angular frequency where $\phi=-\pi / 2$, which means that the phase of the transmission coefficient is also $\phi_{21}=-\pi / 2$ and can be easily computed. Thus, from (A1), (A2) and (A4) we can determine the three reactive element values that contribute to the shunt impedance.

The circuit of Fig. 1(b) has an additional parameter $\left(C_{g}\right)$, therefore, we need an additional condition to fully determine the circuit parameters. This condition can be the resonance frequency of the series branch

$$
f_{s}=\frac{1}{2 \pi \sqrt{L C_{g}}}
$$

which is given by the intercept of $S_{11}$ with the unit conductance circle, where the impedance of the series branch nulls.

\section{APPENDIX B}

\section{INITIAL LINE AND GAP GEOMETRY}

Inversion of expressions (1) and (2) gives:

$$
\begin{gathered}
C_{s}=\frac{4 C_{g}^{2}}{C+4 C_{g}} \\
C_{p a r}=\frac{2 C C_{g}}{C+4 C_{g}}
\end{gathered}
$$

If we assume that the fringing capacitance is small as compared to the line capacitance, it follows that $C_{L}=C_{p a r}$, and from the value of $L$ and $C_{L}$, we obtain the line impedance according to:

$$
Z_{0}=\sqrt{\frac{L}{C_{L}}}
$$

We use this impedance value to determine the line width, $W$, according to well known expressions (the line width is inferred by means of a transmission line calculator). From the substrate parameters and the line width, the gap space, $s$, necessary to obtain the value of $C_{s}$ given by expression (B1) can be inferred according to well known expressions [44]. Finally, the line length, $l$, is inferred by inverting the phase velocity given by:

$$
v_{p}=\frac{c}{\sqrt{\varepsilon_{e f f}}}=\frac{l}{\sqrt{L C_{L}}}
$$

where $c$ is the speed of light in vacuum and $\varepsilon_{\text {eff }}$ is the effective dielectric constant (that can be easily computed from a transmission line calculator).

\section{REFERENCES}

[1] D.R. Smith, W.J. Padilla, D.C. Vier, S.C. Nemat-Nasser and S. Schultz, "Composite medium with simultaneously negative permeability and permittivity”, Phys. Rev. Lett., vol. 84, pp. 4184-4187, 2000.

[2] A. K. Iyer and G. V. Eleftheriades. "Negative refractive index metamaterials supporting 2-D waves," in IEEE-MTT Int'l Microwave Symp., vol. 2, Seattle, WA, pp. 412-415, June 2002.
[3] A.A. Oliner, "A periodic-structure negative-refractive-index medium without resonant elements," in URSI Digest, IEEE-AP-S USNC/URSI National Radio Science Meeting, San Antonio, TX, pp. 41, June 2002.

[4] C. Caloz and T. Itoh. "Application of the transmission line theory of left-handed $(\mathrm{LH})$ materials to the realization of a microstrip $\mathrm{LH}$ transmission line," in Proc. IEEE-AP-S USNC/URSI National Radio Science Meeting, vol. 2, San Antonio, TX, pp. 412-415, June 2002.

[5] F. Martín, F. Falcone, J. Bonache, R. Marqués and M. Sorolla, "Split ring resonator based left handed coplanar waveguide", Appl. Phys. Lett., vol. 83, pp. 4652-4654, December 2003.

[6] F. Falcone, T. Lopetegi, M.A.G. Laso, J.D. Baena, J. Bonache, R. Marqués, F. Martín, M. Sorolla, "Babinet principle applied to the design of metasurfaces and metamaterials", Phys. Rev. Lett., vol. 93, paper 197401, November 2004.

[7] A. Görür, "A Novel Coplanar Slow-Wave Structure", IEEE Microwave and Guided Wave Letters, vol. 4, no. 3, pp. 86-88, March 1994.

[8] J. Sor, Y. Qian, and T. Itoh, "Miniature low-loss CPW periodic structures for filter applications", IEEE Transactions on Microwave Theory and Techniques, vol. 49, no. 12, pp. 2336-2341, December 2001.

[9] C.-W. Wang, T.-G. Ma, and C.-F. Yang, "A new planar artificial transmission line and its applications to a miniaturized butler matrix", IEEE Transactions on Microwave Theory and Techniques, vol. 55, no. 12, pp. 2792-2801, December 2007.

[10] M.A. Antoniades and G.V. Eleftheriades, "A broadband series power divider using zero-degree metamaterial phase shifting lines", IEEE Microwave and Wireless Components Letters, vol. 15, pp.808-810, November 2005.

[11] M. Gil, J. Bonache, I. Gil, J. García-García and F. Martín, "Miniaturization of planar microwave circuits by using resonant-type left handed transmission lines", IET Microwaves Antennas and Propagation, vol. 1 (1), pp. 73-79, Feb. 2007.

[12] H. Okabe, C. Caloz and T. Itoh, "A compact enhanced bandwidth hybrid ring using an artificial lumped element left handed transmission line section", IEEE Trans. Microwave Theory and Techniques, vol. 52, pp. 798-804, March 2004.

[13] M.A. Antoniades and G.V. Eleftheriades, "A broadband Wilkinson balun using microstrip metamaterial lines", IEEE Antennas and Wireless Propagation Lett., vol. 4, pp. 209-212, 2005.

[14] D. Kholodnyak, E. Serebryakova, I Vendik, O. Vendik, "Broadband digital phase shifter based on switchable right- and left-handed transmission line sections", IEEE Microwave Wireless Comp. Lett., vol. 16, 258-260, May 2006.

[15] G. Sisó, M. Gil, J. Bonache and F. Martín, “Application of metamaterial transmission lines to the design of quadrature phase shifters", Electronics Letters, vol. 43, pp. 1098-1100, September 2007.

[16] G. Sisó, J. Bonache, M. Gil and F. Martín, "Application of resonanttype metamaterial transmission lines to the design of enhanced bandwidth components with compact dimensions", Microwave and Optical Technology Letters, vol. 50, pp. 127-134, January 2008.

[17] I.H. Lin, M. De Vincentis, C. Caloz and T. Itoh, "Arbitrary dual-band components using composite right/left handed transmission lines", IEEE Trans. Microwave Theory and Techniques, vol. 52, pp. 1142-1149, April 2004.

[18] S.H. Ji, C.S. Cho, J.W. Lee, and J. Kim, "Concurrent dual-band class-E power amplifier using composite right/left-handed transmission lines", IEEE Transactions on Microwave Theory and Techniques, vol. 55, pp. 1341-1347, June 2007.

[19] J. Bonache, G. Sisó, M. Gil, A. Iniesta, J. García-Rincón and F. Martín, "Application of composite right/left handed (CRLH) transmission lines based on complementary split ring resonators (CSRRs) to the design of dual band microwave components", IEEE Microwave and Wireless Components Letters, vol. 18, pp. 524-526, August 2008.

[20] A. C. Papanastasiou, G. E. Georghiou, and G. V. Eleftheriades, "A quad-band wilkinson power divider using generalized NRI transmission lines", IEEE Microwave and Wireless Components Letters, vol. 18, pp. 521-523, August 2008.

[21] M. Durán-Sindreu, G. Sisó, J. Bonache and F. Martín, "Planar multiband microwave components based on the generalized composite right/left handed transmission line concept", IEEE Transactions on Microwave Theory and Techniques, vol. 58, no 12, pp. 3882-3891, Dec. 2010.

[22] S. Lim, C. Caloz, and T. Itoh, "Metamaterial-based electronicallycontrolled transmission line structure as a novel leaky-wave antenna 
with tunable angle and beamwidth," IEEE Trans. Microwave Theory Techniques, vol. 52, No. 12, pp. 2678-2690, December 2004.

[23] F.P. Casares-Miranda, C. Camacho-Penalosa, C. Caloz, "High-gain active composite right/left-handed leaky-wave antenna", IEEE Transactions on Antennas and Propagation, vol. 54, no.8, pp.22922300, August 2006

[24] J. Bonache, I. Gil, J. García-García, F. Martín, "Novel microstrip band pass filters based on complementary split ring resonators", IEEE Transactions on Microwave Theory and Techniques, vol. 54, pp. 265271, January 2006

[25] M. Gil, J. Bonache, J. García-García, J. Martel and F. Martín, "Composite right/left handed (CRLH) metamaterial transmission lines based on complementary split rings resonators (CSRRs) and their applications to very wide band and compact filter design", IEEE Transactions on Microwave Theory and Techniques, vol. 55, pp. 12961304, June 2007.

[26] M. Durán-Sindreu, A. Vélez, G. Sisó, J. Selga, P. Vélez, J. Bonache, and F. Martín, "Recent advances in metamaterial transmission lines based on split rings", Proceedings of the IEEE, vol. 99, pp. 1701-1710 October 2011.

[27] D Bouyge, D. Mardivirin, J. Bonache, A. Crunteanu, A. Pothier, M. Durán-Sindreu, P. Blondy, F. Martín, "Split ring resonators (SRRs) based on micro-electro-mechanical deflectable cantilever-type rings: application to tunable stopband filters", IEEE Microwave and Wireless Components Letters, vol. 21, pp. 243-245, May 2011.

[28] C. Caloz, and T. Itoh, Electromagnetic Metamaterials: Transmission Line Theory and Microwave Applications, John Wiley \& Sons, Inc., USA, 2006.

[29] R. Marques, F. Martín, and M. Sorolla, Metamaterials with Negative Parameters: Theory, Design and Microwave Applications, John Wiley \& Sons, Inc., USA, 2008.

[30] J.B. Pendry, A.J. Holden, D.J. Robbins and W.J. Stewart, "Magnetism from conductors and enhanced nonlinear phenomena", IEEE Transactions Microwave Theory Techn., vol. 47 pp. 2075-2084, Nov. 1999.

[31] F. Falcone, T. Lopetegi, J.D. Baena, R. Marqués, F. Martín and M. Sorolla, "Effective negative- $\varepsilon$ stop-band microstrip lines based on complementary split ring resonators", IEEE Microwave and Wireless Components Letters, vol. 14, pp. 280-282, June 2004.

[32] J. Bonache, M. Gil, O. García-Abad and F. Martín, "Parametric analysis of microstrip lines loaded with complementary split ring resonators," Microw. Opt. Techn. Lett., vol. 50, pp. 2093-2096, August 2008.

[33] J. García-García, F. Martín, F. Falcone, J. Bonache, I. Gil, T. Lopetegi, M.A.G. Laso, M. Sorolla, R. Marqués, "Spurious passband suppression in microstrip coupled line band pass filters by means of split ring resonators", IEEE Microwave and Wireless Components Letters, vol. 14, pp. 416-418, September 2004.

[34] J. Selga, A. Rodríguez, M. Gil, J. Carbonell, V.E. Boria, and F. Martín "Synthesis of Planar Microwave Circuits through Aggressive Space Mapping using commercially available software packages", Int. J. RF Microw. Computer-Aided Engineering, vol. 20 (5), pp. 527-534 September 2010

[35] J. Selga, A. Rodríguez, M. Gil, J. Carbonell, J. Bonache V.E. Boria, and F. Martín, "Towards the Automatic Layout Synthesis in Resonant-type Metamaterial Transmission Lines", IET Microwaves Antennas and Propagation, vol. 4(8), pp. 1007-1015, Aug. 2010.

[36] J.W. Bandler, R.M. Biernacki, S.H. Chen, P.A. Grobelny and R.H Hemmers, "Space mapping technique for electromagnetic optimization", IEEE Transactions on Microwave Theory and Techniques, vol. 42, pp. 2536-2544, Dec. 1994

[37] J.W. Bandler, R.M. Biernacki, S.H. Chen, R.H. Hemmers, and K. Madsen, "Electromagnetic optimization exploiting aggressive space mapping", IEEE Transactions on Microwave Theory and Techniques, vol. 43, pp. 2874-25442882, Dec. 1995.

[38] J. W. Bandler, Q. S. Cheng, S. A. Dakroury, A. S. Mohamed, M. H. Bakr, K. Madsen, and J. Søndergaard, "Space mapping: the state of the art," IEEE Trans. Microwave Theory Tech., vol. 52, pp. 337-361, 2004

[39] J. Selga, A. Rodriguez, V. E. Boria and F. Martín, "Application of Aggressive Space Mapping to the Synthesis of Composite Right/Left Handed (CRLH) Transmission Lines Based on Complementary Split Ring Resonators," European Microwave Conf. Dig., pp. 968-971, Oct 2011.

[40] D.M. Bates, D.G. Watts, Nonlinear regression analysis and its applications, John Wiley, New York, 1998.
[41] MATLAB version 2010a, The MathWorks Inc., 2010.

[42] Agilent Momentum version 8.20.374, Agilent Technologies, 2008.

[43] Ansoft Designer version 6.1.0, Ansys Inc., 2010

[44] T.C. Edwards, M.B. Steer, Foundations of interconnect and microstrip design, $3^{\text {rd }}$ Ed., John Wiley, New York, 2000

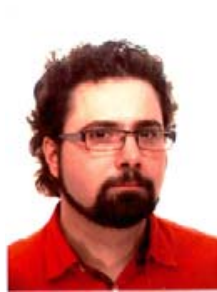

Jordi Selga was born in Barcelona, Spain, in 1982. He received the Telecommunications Engineering Diploma in 2006 and the Electronics Engineering degree in 2008 from the Universitat Autònoma de Barcelona (UAB), Barcelona, Spain. In 2008 he joined CIMITEC-UAB, a research centre on Metamaterials supported by TECNIO (Catalan Government). He obtained the Master degree in Micro and Nano Electronics Engineering in 2009 from UAB, and he is currently working towards his $\mathrm{PhD}$ degree in subjects related to metamaterials, CAD design of microwave devices and EM optimization methods at the UAB. He was holder of a national research fellowship from the Formación de Profesorado Universitario Program of the Education and Science Ministry (Reference AP2008-4707).

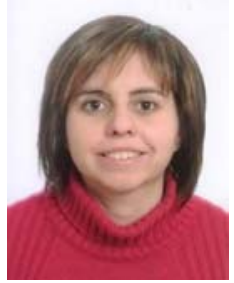

Ana Rodriguez was born in Lugo, Spain. She received the Telecommunications Engineering degree from the Universidade de Vigo (UV), Spain, in 2008. As a student, she participated in the Erasmus exchange program, developing the Master Thesis at the University of Oulu, Finland. Since the end of 2008, she has joined the Institute of Telecommunications and Multimedia Applications (iTEAM), which is part of the scientific park at the Universitat Politècnica de València (UPV), Spain. She obtained "Master en Tecnología, Sistemas y Redes de Comunicaciones" in 2010 from UPV, and currently is on the way of pursuing Ph.D. degree. Her main research interests include CAD design of microwave devices, EM optimization methods and metamaterials.

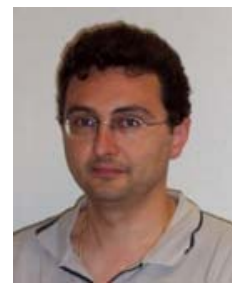

Vicente E. Boria (S'91-A'99-SM'02) was born in Valencia, Spain, on May 18, 1970. He received his "Ingeniero de Telecomunicación" degree (with firstclass honors) and the "Doctor Ingeniero de Telecomunicación" degree from the Universidad Politécnica de Valencia, Valencia, Spain, in 1993 and 1997, respectively. In 1993 he joined the "Departamento de Comunicaciones", Universidad Politécnica de Valencia, where he has been Full Professor since 2003. In 1995 and 1996, he was holding a Spanish Trainee position with the European Space Research and Technology Centre, European Space Agency (ESTEC-ESA), Noordwijk, The Netherlands, where he was involved in the area of EM analysis and design of passive waveguide devices. He has authored or co-authored 7 chapters in technical textbooks, 75 papers in refereed international technical journals, and over 150 papers in international conference proceedings. His current research interests are focused on the analysis and automated design of passive components, left-handed and periodic structures, as well as on the simulation and measurement of power effects in passive waveguide systems.

Dr. Boria has been a member of the IEEE Microwave Theory and Techniques Society (IEEE MTT-S) and the IEEE Antennas and Propagation Society (IEEE AP-S) since 1992. He is member of the Editorial Boards of the IEEE Transactions on Microwave Theory and Techniques, IEEE Microwave and Wireless Components Letters, Proceeding of the IET (Microwaves, Antennas and Propagation), IET Electronics Letters and Radio Science. He is also a member of the Technical Committees of the IEEE-MTT International Microwave Symposium and of the European Microwave Conference.

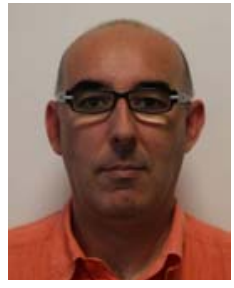

Ferran Martín (M'04-SM'08-F'12) was born in Barakaldo (Vizcaya), Spain in 1965 . He received the B.S. Degree in Physics from the Universitat Autònoma de Barcelona (UAB) in 1988 and the $\mathrm{PhD}$ degree in 1992. From 1994 up to 2006 he was Associate Professor in Electronics at the Departament d'Enginyeria Electrònica (Universitat Autònoma de Barcelona), and from 2007 he is Full Professor of Electronics. In recent years, he has been involved in different research activities including modelling and simulation of electron devices for high frequency applications, millimeter 
wave and $\mathrm{THz}$ generation systems, and the application of electromagnetic bandgaps to microwave and millimeter wave circuits. He is now very active in the field of metamaterials and their application to the miniaturization and optimization of microwave circuits and antennas. He is the head of the Microwave and Millimeter Wave Engineering Group (GEMMA Group) at UAB, and director of CIMITEC, a research Center on Metamaterials supported by TECNIO (Generalitat de Catalunya). He has organized several international events related to metamaterials, including Workshops at the IEEE International Microwave Symposium (years 2005 and 2007) and European Microwave Conference (2009). He has acted as Guest Editor for three Special Issues on Metamaterials in three International Journals. He has authored and co-authored over 350 technical conference, letter and journal papers and he is co-author of the monograph on Metamaterials entitled Metamaterials with Negative Parameters: Theory, Design and Microwave Applications (John Wiley \& Sons Inc.). Ferran Martín has filed several patents on metamaterials and has headed several Development Contracts. Among his distinctions, Ferran Martín has received the 2006 Duran Farell Prize for Technological Research, he holds the Parc de Recerca UAB Santander Technology Transfer Chair, and he has been the recipient of an ICREA ACADEMIA Award. 